\title{
FROM INDIVIDUAL TO COLLECTIVE BEHAVIOR IN BACTERIAL CHEMOTAXIS
}

\author{
RADEK ERBAN* AND HANS G. OTHMER ${ }^{\dagger}$
}

\begin{abstract}
Bacterial chemotaxis is widely studied from both the microscopic (cell) and macroscopic (population) points of view, and here we connect these different levels of description by deriving the classical macroscopic description for chemotaxis from a microscopic model of the behavior of individual cells. The analysis is based on the velocity jump process for describing the motion of individuals such as bacteria, wherein each individual carries an internal state that evolves according to a system of ordinary differential equations forced by a time- and/or space-dependent external signal. In the problem treated here the turning rate of individuals is a functional of the internal state, which in turn depends on the external signal. Using moment closure techniques in one space dimension, we derive and analyze a macroscopic system of hyperbolic differential equations describing this velocity jump process. Using a hyperbolic scaling of space and time we obtain a single second-order hyperbolic equation for the populations density, and using a parabolic scaling we obtain the classical chemotaxis equation, wherein the chemotactic sensitivity is now a known function of parameters of the internal dynamics. Numerical simulations show that the solutions of the macroscopic equations agree very well with the results of Monte Carlo simulations of individual movement.
\end{abstract}

1. Introduction. The ability to detect and respond to changes in the environment is a basic necessity for survival of all organisms, and as a result, a variety of mechanisms have evolved by which organisms sense their environment and respond to signals they detect. Often the response involves movement toward a more favorable environment or away from a noxious substance. The movement response can entail changing the speed of movement and the frequency of turning, which is called kinesis, it may involve directed movement, which is called taxis, or it may involve a combination of these. Taxes and kineses may be characterized as positive or negative, depending on whether they lead to accumulation at high or low points of the external stimulus that triggers the motion. A variety of both modes are known, and include responses to gradients of oxygen and other chemicals, gradients of adhesion to the substrate, and others. Both tactic and kinetic responses involve two major steps: (i) detection of the signal, and (ii) transduction of the external signal into an internal signal that triggers the response. From the modeling and analysis standpoint, an important characteristic of both modes of response is whether or not the individual merely detects the signal or alters it as well, for example by amplifying it so as to relay the signal. When there is no significant alteration the individual simply responds to the spatio-temporal distribution of the signal. However when the individual produces or degrades the signal, there is coupling between the local density of individuals and the intensity of the signal. This occurs, for example, when individuals aggregate in response to a signal from 'organizers' and relay the signal as well.

In several systems, including the flagellated bacterium E. coli and the amoeboid cell Dictyostelium discoideum, a detailed understanding of how extracellular signals are transduced into behavioral changes is emerging from experimental work, while at the macroscopic level a great deal is known about solutions of the classical chemotaxis equations. However, the chemotaxis equations to date have been based on phenomenological descriptions of how cells respond to signals, and at present there is little understanding of how microscopic properties translate into the macroscopic parameters. The motion of E. coli has been studied for forty years and much is known about how they sense and process environmental signals. E. coli alternates two basic behavioral modes, a more or less linear motion called a run, and a highly erratic motion called tumbling, the purpose of which is to reorient the cell. During a run the bacteria move at approximately constant speed in the most recently chosen direction. Run times are typically much longer than the time spent tumbling, and when bacteria move in a favorable direction (i.e., either in the direction of foodstuffs or away from harmful substances) the run times are increased further. These bacteria are too small to detect spatial differences in the concentration of an attractant on the scale of a cell length, and during a tumble they simply choose a new direction essentially at random, although it has a some bias in the direction of the preceding run [7, 4]. The effect of alternating these two modes of behavior, and in particular, of increasing the run length when moving in a favorable direction, is that a bacterium executes a three-dimensional random walk with drift in a favorable direction when observed on a sufficiently long time scale [4, 24, 5]. Models for signal transduction and adaptation in this system are given in [40, 2, 27].

\footnotetext{
* School of Mathematics, 270B Vincent Hall, University of Minnesota, Minneapolis, MN 55455 (erban@math.umn.edu). Research supported in part by NSF grant DMS 0317372 .

† School of Mathematics, 270A Vincent Hall, University of Minnesota, Minneapolis, MN 55455 (othmer@math.umn.edu). Research supported in part by NIH grant GM 29123, NSF grant DMS 9805494 and NSF grant DMS 0317372.
} 
In the absence of external ques, many organisms use a random walk strategy to determine their pattern of movement. In this case the movement of organisms released at a point in a uniform environment can be described as an uncorrelated, unbiased random walk of noninteracting particles on a sufficiently long time scale. In an appropriate continuum limit the cell density $n$, measured in units of cells/ $/{ }^{N}$, where $\mathrm{L}$ denotes length and $\mathrm{N}=1,2$ or 3 , satisfies the diffusion equation

$$
\frac{\partial n}{\partial t}=D \Delta n
$$

Here the cell flux is given by $j=-D \nabla n$, and the simplest description of cell motion in the presence of an attractant or repellent is obtained by adding a directed component to the diffusive flux to obtain

$$
j=-D \nabla n+n u_{c},
$$

where $\mathrm{u}_{c}$ is the macroscopic chemotactic velocity. The taxis is positive or negative according as $u_{c}$ is parallel or anti-parallel to the direction of increase of the chemotactic substance. The resulting evolution equation for $n$ is

$$
\frac{\partial n}{\partial t}=\nabla \cdot\left(D \nabla n-n u_{c}\right),
$$

and this is called a chemotaxis equation. In a phenomenological approach one postulates a constitutive relation for the chemotactic velocity of the form

$$
u_{c}=\chi(S) \nabla S,
$$

where $S$ is the concentration of the chemotactic substance and the function $\chi(S)$ is called the chemotactic sensitivity. When $\chi>0$ the tactic component of the flux is in the direction of $\nabla S$ and the taxis is positive. With this postulate (1.3) takes the form

$$
\frac{\partial n}{\partial t}=\nabla \cdot(D \nabla n-n \chi(S) \nabla S)
$$

We call equations of this type classical chemotaxis equations, though frequently that term is used for a system of equations comprising (1.5) and a reaction-diffusion equation for the evolution of the signal substance. A recent review of the mathematical aspects of chemotaxis equations is given in [20].

A problem in using equations such as (1.5) to describe chemotaxis is how one justifies the constitutive assumption (1.4), and in particular, how one incorporates microscopic responses of individual cells into the chemotactic sensitivity. A number of phenomenological approaches to the derivation of the chemotactic sensitivity or chemotactic velocity have been taken, including simply postulating the form in $(1.4)[22,33]$, or deriving the velocity directly in terms of forces exerted by the cell [35]. Other more fundamental approaches have also been used to relate the chemotactic velocity or sensitivity to a microscopic description of movement. In the first one begins with a lattice walk or space jump process, either in discrete or continuous time, and postulates how the transition probabilities depend on the external signal. For a discrete time walk the chemotaxis equation is derived in the diffusion limit of this process, by letting the space step size $h$ and the time step $\delta t$ go to zero in such a way that the ratio $h^{2} / \delta t$ is a constant, namely $D$. A more general approach leads to a renewal equation, from which a partial differential equation is obtained by particular choices of the jump kernel and the waiting time distribution [28]. Another method, based on a continuous time reinforced random walk in which the walker modifies the transition probabilities of an interval for successive crossings, is developed in [32] for a single tactic substance.

A space-jump process is suitable for certain organisms, but an alternative stochastic process that may be more appropriate to describe the motion of cells is called the velocity-jump process [28]. In this process the velocity, rather than the spatial position, changes by random jumps at random instants of time. The governing evolution equation for the simplest version of this process is

$$
\frac{\partial}{\partial t} p(x, v, t)+v \cdot \nabla p(x, v, t)=-\lambda p(x, v, t)+\lambda \int_{V} T\left(v, v^{\prime}\right) p\left(x, v^{\prime}, t\right) d v^{\prime}
$$

where $p(x, v, t)$ denotes the density of particles at spatial position $x \in \Omega \subset R^{N}$, moving with velocity $v \in V \subset R^{N}$ at time $t \geq 0$ [28]. Here $\lambda$ is the (constant) turning rate and $1 / \lambda$ is a measure of the mean run length between velocity jumps. In general, the turning frequency $\lambda$ must depend on the extracellular signal, as transduced through the signal transduction 
network and the motility control system. The turning kernel $T\left(v, v^{\prime}\right)$ gives the probability of a velocity jump from $v^{\prime}$ to $v$ if a jump occurs, and implicit in the above formulation is the assumption that the choice of a new velocity is independent of the run length.

The forward equation (1.6) for a velocity jump process is similar to the Boltzmann equation, wherein the right hand side is an integral operator that describes the collision of two particles, and is therefore quadratic in $p$ [11]. The kernel of the integral operator is specified by the dynamics, and it is well known that an appropriate scaling of space and time leads at least formally from the Boltzmann equation to a diffusion process [25, 15]. This also holds for transport equations and more general transport processes (see e.g. $[16,34,36]$ ). The earliest derivation of the chemotactic sensitivity from a velocity jump process was done by Patlak [36], who used kinetic theory arguments to express $u_{c}$ in terms of averages of the velocities and run times of individual cells. Alt [1] has used Patlak's approach to relate the chemotactic velocity to the gradient of an attractant or repellent.

In $[18,29]$ the kinetic equation approach for deriving chemotactic equations was further developed using a kernel $T$ that may include an external bias. A general Perron-Frobenius property of the turning operator $\mathcal{T}$ defined by the right-hand side of (1.6) and a proper scaling of space and time lead to a Hilbert expansion of the long-term dynamics that produces a parabolic limiting equation. In certain cases there is no taxis and the parabolic limit is anisotropic, in that the resulting equation for the macroscopic density

$$
n(x, t)=\int_{V} p(x, v, t) \mathrm{d} v
$$

is

$$
\frac{\partial n}{\partial t}=\nabla \cdot D \nabla n,
$$

where $D$ is an $N \times N$ non-diagonal matrix. Necessary and sufficient conditions under which the diffusion matrix $D$ reduces to a scalar times the identity were also obtained. In previous work the external bias enters the turning kernel and turning rate as an order $\varepsilon$ term [1,29], and the perturbation analysis done in [29] shows that the chemotaxis equation is obtained only in this case. In the approach used in [29] an external bias of order one in the turning kernel can be admitted, but with suitable restrictions this leads to (1.8) rather than the chemotaxis equation in the diffusion limit.

The prototypical organisms whose motion can be described as a velocity jump process are the flagellated bacteria such as E. coli. A bacterium runs at a constant velocity for a random length of time, then tumbles for a random length of time, chooses a new direction at random, and repeats the cycle. When motion is restricted to one space dimension and the tumble phase is neglected this leads to a telegraph process described by the hyperbolic system

$$
\begin{aligned}
& \frac{\partial p^{+}}{\partial t}+s \frac{\partial p^{+}}{\partial x}=-\lambda p^{+}+\lambda p^{-} \\
& \frac{\partial p^{-}}{\partial t}-s \frac{\partial p^{-}}{\partial x}=\lambda p^{+}-\lambda p^{-}
\end{aligned}
$$

where $p^{ \pm}(x, t)$ are the probability density of particles that are at $(x, t)$ and are moving to the right $(+)$ and left $(-)$, and $s$ is the speed. This model was first analyzed by Goldstein [14], and subsequently by others [21, 26, 28]. It can be shown that on a finite domain with reflecting boundary conditions, with $\lambda$ a constant, the system reduces to a damped wave equation called the telegraph equation for the total density $p \equiv p^{+}+p^{-}$, and solutions are asymptotically constant in space and time. Even if there is a fixed background signal and the turning rate depends on the signal but is independent of the direction of travel, still there is no aggregation at extrema of the signal: all solutions are asymptotically constant [31].

It is not difficult to see formally that the turning rate for left-moving particles must be different from that for rightmoving particles in order to produce aggregation, and this has been analyzed in detail in [19]. However, at present there is little understanding of the interplay between the intracellular dynamical system that describes signal transduction and quantities such as the turning rate and turning kernel in a macroscopic, population-level description of motion. Our objective here is to develop a mathematical framework in which one can systematically extract information about population-level behavior for adapting walkers from microscopic models of individual behavior, and to apply it to a caricature of adapting intracellular dynamics. We use E. coli as a prototype system, but the methodology and the results apply more generally. 
The paper is organized as follows. In the following section we briefly describe the signal transduction network in $E$. coli to motivate a simplified description used herein. Next we introduce the transport equations for systems with internal dynamics, which are the starting point for the derivation of the macroscopic limit. We derive the macroscopic moment equations (Section 4), the modified version of the classical chemotaxis equation (Section 6) and the classical chemotaxis equation (Section 7). Finally, we show some illustrative numerical results, we apply our results to the experiments with the exponential signal gradient and we discuss generalizations of our approach.

2. Internal dynamics. E. coli have 6-8 flagella distributed uniformly over the cell surface and move by rotating them in a corkscrew-like manner [37, 41]. When rotated counterclockwise, the flagella coalesce into a propulsive bundle, resulting in a relatively straight "run" [8]. When rotated clockwise they fly apart, resulting in a "tumble" which reorients the cell but causes no significant change of location. The cell thus alternates between runs and reorienting tumbles. In the absence of stimuli, the bias or probability per unit time of a tumble $\left(P_{C W}\right)$ is essentially independent of when the last tumble occurred [41]. The mean run interval is about $1 \mathrm{sec}$ in the absence of chemotaxis, the mean tumble interval is about $0.1 \mathrm{sec}$, and both are distributed exponentially [6]. A chemoeffector (attractant or repellent) alters the probabilities that the flagella will rotate in a given direction, thus changing the frequencies and duration of runs and tumbles. E. coli respond chemotactically to a variety of attractants and repellents over a wide range of concentrations [6]. A typical response, which we define as a measurable change in bias from baseline following a transient increase in the concentration of an attractant or a decrease in that of a repellent, is as follows. After a brief latency period there is an increase in $\mathrm{P}_{C C W}$ (probability per unit time of a run) above the baseline probability of approximately 0.64 [8]. This early response, which is typically rapid, constitutes the excitation, and it is followed by a period of relatively slow adaptation to the stimulus. Adaptation eventually returns the bias to baseline, allowing the cell to respond to further changes.

The magnitude of the change in bias in response to an exponentially-increasing attractant concentration increases approximately linearly with the ramp rate [9]. Assuming equilibrium binding, the fraction of receptors occupied is $\theta=$ $S /\left(K_{D}+S\right)$, where $S$ is the concentration and $K_{D}$ is the dissociation constant. Therefore $\dot{\theta}=K_{D} S /\left(K_{D}+S\right)^{2} \cdot d \ln S / d t$, and if $S \sim K_{D}$, then $4 \dot{\theta} \sim d \ln S / d t$, which is the ramp rate. Thus the magnitude of the response is an approximately linear function of the rate of change in occupancy, which provides a superficial explanation of the observed adaptation. Because of adaptation, the response is not directly dependent on the absolute concentration of chemoeffector [41], but instead the sensory system functions as a derivative detector.

E. coli is also extremely sensitive to small changes in chemoeffector levels. The cells can respond to slow exponential increases in attractant levels that correspond to rates of change in the fractional occupancy of chemoreceptors as small as $0.1 \%$ per second $[9,38]$. Thus a cell can respond even when there is only a small change in the receptor occupancy over a typical sampling period. High sensitivity is also seen when cells are subjected to small impulses or step increases in attractant concentration, though the evidence is mixed. [38] report that a change in receptor occupancy of $0.42 \%$ elicits a $23 \%$ change in bias - a ratio, or gain, of 55 - but [23] report a maximum gain of only 6 .

The main features of the E. coli chemotaxis excitation and adaptation pathways are as follows [10] (see Figure 2.1). Chemical stimuli are detected by transmembrane receptors, which in turn generate cytoplasmic signals that control the flagellar motors. Aspartate, the attractant chemoeffector most commonly used in experiments, binds directly to the periplasmic domain of its transducer, Tar. This initiates a complicated sequence of biochemical steps, the net effect of which is to temporarily reduce the level of $\mathrm{CheY}_{P}$ following an increase in attractant, thereby temporarily increasing $P_{C C W}=1-P_{C W}$ and increasing the fraction of time spent running as opposed to tumbling. Detailed models of this network are now available $[40,2,27]$, and we refer the reader to the original literature. For our purposes we wish to abstract the essential features of the signal transduction and response processes.

2.1. Cartoon internal dynamics. The essential aspects that a simplified description must reproduce in order to make it useful for studying macroscopic phenomena are (i) it must exhibit excitation, which here means a change in bias in response to a stimulus, (ii) the bias must return to baseline levels (i.e., the response must adapt) on a time scale that is slow compared to excitation, and (iii) the signal transduction network should amplify signals appropriately. Let $y=$ $\left(y_{1}, y_{2}, \ldots, y_{m}\right) \in \mathbb{R}^{m}$ denote the internal state variables, which can include the concentration of receptors, proteins, etc., and let $S(x, t)=\left(S_{1}, S_{2}, \ldots, S_{M}\right) \in \mathbb{R}^{M}$ denote the signals in the environment. Then all present deterministic models of bacterial signal transduction pathways can be cast in the form of a system of ordinary differential equations that describe the evolution of the intracellular state, forced by the extracellular signal. Thus

$$
\frac{\mathrm{d} y}{\mathrm{~d} t}=f(y, S)
$$




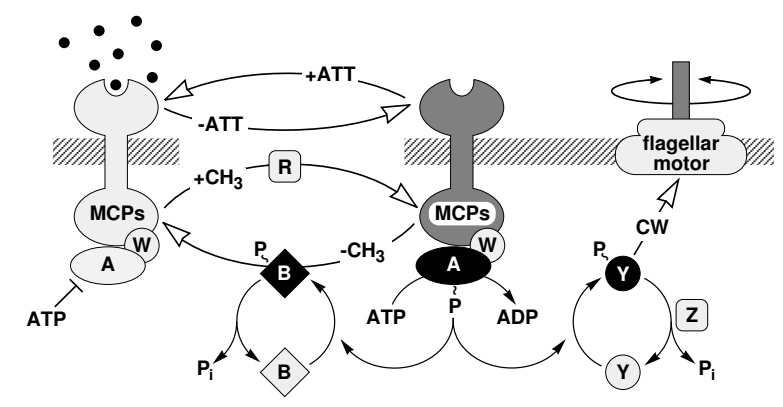

FIG. 2.1. Signaling components and pathways for E. coli chemotaxis. Chemoreceptors (MCPs) span the cytoplasmic membrane (hatched lines), with a ligand-binding domain on the outside and a catalytic domain on the cytoplasmic side. MCP complexes have two alternative signaling states. In the attractant-bound form, the receptor inhibits CheA autokinase activity; in the unliganded form, the receptor stimulates CheA (A) activity. The overall flux of phosphoryl groups $P$ to $C h e B(B)$ and Che $Y(Y)$ reflects the proportion of signaling complexes in the inhibited and stimulated states. Changes in attractant concentration shift this distribution, triggering a flagellar response. Adaptation occurs when the ensuing changes in CheB phosphorylation state alter its methylesterase activity, producing a net change in MCP methylation state that cancels the stimulus signal (cf [42] for a review; figure reproduced from [40], with permission).

where $f: \mathbb{R}^{m} \times \mathbb{R}^{M} \rightarrow \mathbb{R}^{m}$ describes the particular model. Now if we have a good microscopic model, can we derive a macroscopic description, and can we use it to predict the effect on macroscopic behavior of changes in the microscopic parameters? At present this is very difficult to do with a full description of the internal dynamics for $E$. coli, which may involve 20 or more variables, and as a first step we use a simpler cartoon description of signal transduction that was developed in [31], and which incorporates the essential features described above.

We describe the internal dynamics with two internal variables, i.e., $y \in Y \subset \mathbb{R}^{2}$, and suppose that internal state evolves according to the system of ordinary differential equations

$$
\begin{gathered}
\frac{\mathrm{d} y_{1}}{\mathrm{~d} t}=\frac{g(S(x))-\left(y_{1}+y_{2}\right)}{t_{e}}, \\
\frac{\mathrm{d} y_{2}}{\mathrm{~d} t}=\frac{g(S(x))-y_{2}}{t_{a}},
\end{gathered}
$$

where $t_{e}$ and $t_{a}$ are constants, $x$ is the current position of a cell, $S: \mathbb{R}^{N} \times[0, \infty) \rightarrow[0, \infty)$ is the concentration of the chemoattractant, and $g:[0, \infty) \rightarrow[0, \infty)$ models the first step of signal transduction. For any constant signal $S$ these equations have the property that

$$
\lim _{t \rightarrow \infty} y_{1}=0 \text { and } \lim _{t \rightarrow \infty} y_{2}=g(S)
$$

and therefore $y_{1}$ adapts perfectly to any constant stimulus. The time constants $t_{e}$ and $t_{a}$ are labeled in anticipation of using $y_{1}$ for the internal response, and $y_{2}$ as the adaptation variable, and therefore we call $t_{e}$ and $t_{a}$ the excitation and adaptation time constant, respectively. In order to obtain the desired response, one must have $t_{e}<t_{a}$. In E. coli the excitation is much faster than adaptation, and we have $t_{e} \ll t_{a}$.

Since $y_{1}$ adapts perfectly, any continuous function $h: \mathbb{R} \rightarrow \mathbb{R}$ of $y_{1}$ can be used to model the response to changes in the extracellular signal, and the response will adapt, i.e., the steady state response will be independent of the magnitude of the stimulus $S$. In Figure 2.2 we compare the response of the cartoon model with the response predicted by a detailed model of the entire signal transduction pathway. It is clear that the cartoon model can capture the essential changes in the bias in $E$. coli using a suitable definition of the response. For the simplest velocity jump process in which tumbling is ignored, we identify the response with the turning frequency $\lambda(y)$. In a more detailed description in which the tumble phase is accounted for, one can relate the internal state more directly to experimental results on the switching frequency [12]. This will be done in Section 9.1; here we use $\lambda(y) \equiv$ Response $=h\left(y_{1}\right)$. Moreover, for simplicity we will assume that $h$ is a linear function of $y_{1}$ (which is always true for small responses $y_{1}$ ), i.e., we suppose that

$$
\lambda(y) \equiv \text { Response }=\lambda_{0}-b y_{1},
$$



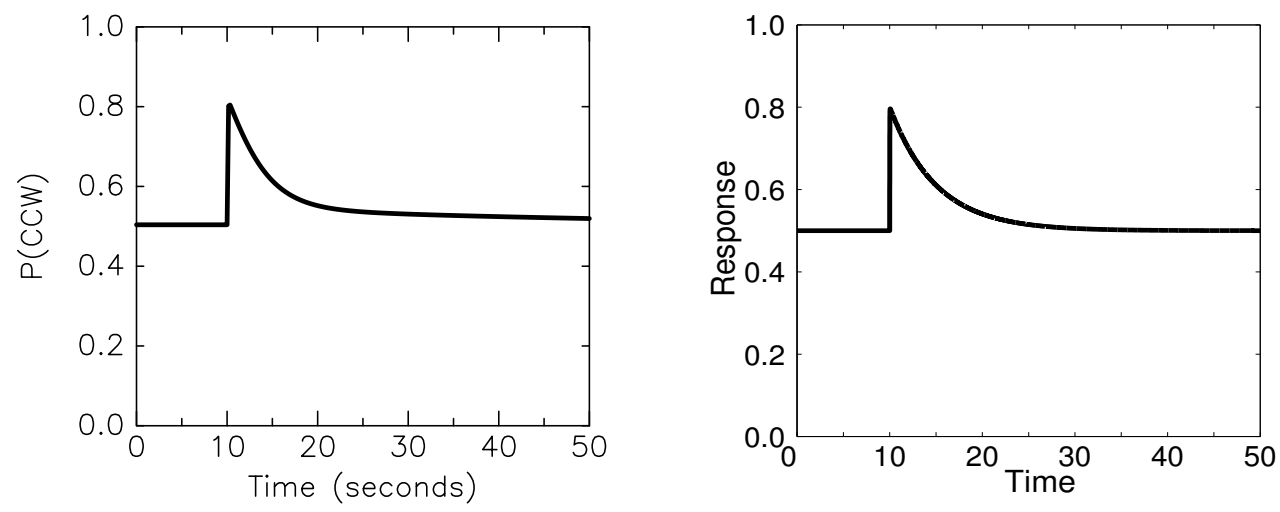

FIG. 2.2. (left) The computed change in bias in response to a step change in attractant for a complete signal transduction model [40]. (right) The graph of the response of the internal dynamics (2.2) - (2.3) given a step change of the signal. Here the response is defined as Response $=0.5+y_{1}$, moreover, $g=$ Identity, $t_{e}=0.01$ and $t_{a}=5$. The signal function is 0 in the time interval $[0,10]$ and the signal is equal to 0.3 in the time interval $[10,50]$.

where $\lambda_{0}$ is the basal turning frequency for a fully-adapted cell and $b$ is a positive constant. The term $b y_{1}$ describes the change in the turning frequency in response to a signal, and negative sign accounts for the fact that an increase of $y_{1}$ should produce a decrease in turning rate.

The function $g$ in (2.2) and (2.3) describes the transduction of the signal, and a reasonable choice for this is to suppose that it depends on the fraction of receptors occupied, in which case

$$
g(S)=G\left(\frac{S}{K_{D}+S}\right)
$$

for some other function $G$, where $K_{D}$ is the dissociation constant for the attractant [9]. However, without loss of generality we shall assume initially that $g=$ Identity. Furthermore, we will initially assume that $t_{e}=0$ in (2.2). The results for the general function $g$ and $t_{e} \neq 0$ can be derived similarly and we state them at the end of the corresponding sections (see (6.25), (7.13) etc.).

Whatever the choice of $g$, the formal solution to (2.2) and (2.3) can be obtained explicitly. However, because $x=x(t)$ is the cell position at time $t$ in a given external concentration field, the integration must be along the cell trajectory, which is a biased random walk. Hence, $S(x, t)$ is a stochastic input to the signal transduction system.

3. Individual behavior. We suppose that the extracellular signal is specified as $S(x, t)$, and for the present we neglect the time spent tumbling. The tumble phase is incorporated in Section 9.1. Let $p(x, v, y, t)$ be the density function of bacteria in a $(2 N+m)$-dimensional phase space with coordinates $(x, v, y)$, where $x \in \mathbb{R}^{N}$ is the position of a cell, $v \in V \subset \mathbb{R}^{N}$ is its velocity and $y \in Y \subset \mathbb{R}^{m}$ is its internal state, which evolves according to (2.1). Thus $p(x, v, y, t) \mathrm{d} x \mathrm{~d} v \mathrm{~d} y$ is the number of cells with position between $x$ and $x+\mathrm{d} x$, velocity between $v$ and $v+\mathrm{d} v$, and internal state between $y$ and $y+\mathrm{d} y$. The evolution of $p$ is governed by the following transport equation

$$
\frac{\partial p}{\partial t}+\nabla_{x} \cdot v p+\nabla_{v} \cdot F p+\nabla_{y} \cdot f p=\mathcal{Q}
$$

where $F$ denotes the external force acting on the individuals and $\mathcal{Q}$ is the rate of change of $p$ due to reactions, random choices of velocity, collisions, etc. Here we ignore external forces and set $F \equiv 0$. Moreover, we assume that there is only one process represented in $\mathcal{Q}$ : that which generates the random velocity change, and we assume that the changes are the result of a Poisson process of intensity $\lambda(y)$. Then

$$
\mathcal{Q}=-\lambda(y) p(x, v, y, t)+\int_{V} \lambda(y) T\left(v, v^{\prime}, y\right) p\left(x, v^{\prime}, y, t\right) \mathrm{d} v^{\prime}
$$

where the kernel $T\left(v, v^{\prime}, y\right)$ gives the probability of a change in velocity from $v^{\prime}$ to $v$, given that a reorientation occurs. The kernel $T$ is non-negative and satisfies the normalization condition $\int_{V} T\left(v, v^{\prime}, y\right) \mathrm{d} v=1$. 
Consequently, the transport equation (3.1) takes the following form

$$
\frac{\partial p}{\partial t}+\nabla_{x} \cdot v p+\nabla_{y} \cdot f p=-\lambda(y) p+\int_{V} \lambda(y) T\left(v, v^{\prime}, y\right) p\left(x, v^{\prime}, y, t\right) \mathrm{d} v^{\prime} .
$$

The objective of this paper is to derive a macroscopic description for chemotaxis from the microscopic model, i.e., an evolution for the macroscopic density of individuals

$$
n(x, t)=\int_{Y} \int_{V} p(x, v, y, t) \mathrm{d} v \mathrm{~d} y
$$

Since we are primarily concerned with how the internal dynamics (2.1) influence the macroscopic behavior, we will only consider movement in 1D. Considering 2D and 3D models does not alter the process of incorporating the internal dynamics into the macroscopic equations, but it does raise technical issues that will be discussed elsewhere. Moreover, we assume that the speed is constant, and therefore we analyze the following generalization of the simple telegraph process described by (1.9): Let $p^{+}(x, y, t)$ be the probability density of the particles that are at $(x, t)$ with the internal state $y$ and are moving to the right, and let $p^{-}(x, y, t)$ be the probability density of the particles that are at $(x, t)$ with the internal state $y$ and are moving to the left. Let the internal state evolve according to the system of ordinary differential equations (2.1). Then $p^{ \pm}(x, y, t)$ satisfy the equations

$$
\begin{gathered}
\frac{\partial p^{+}}{\partial t}+s \frac{\partial p^{+}}{\partial x}+\sum_{i=1}^{m} \frac{\partial}{\partial y_{i}}\left[f_{i}(y, S) p^{+}\right]=\lambda(y)\left[-p^{+}+p^{-}\right] \\
\frac{\partial p^{-}}{\partial t}-s \frac{\partial p^{-}}{\partial x}+\sum_{i=1}^{m} \frac{\partial}{\partial y_{i}}\left[f_{i}(y, S) p^{-}\right]=\lambda(y)\left[p^{+}-p^{-}\right] .
\end{gathered}
$$

To analyze the system (3.4) - (3.5), we must specify the internal dynamics (2.1) and the turning rate $\lambda(y)$. Here we shall use the internal dynamics (2.2) - (2.3), and the turning rate given by (2.5). Moreover, for simplicity we suppose that the signal $S(x)$ is a time-independent scalar function, that $t_{e}=0$ and that $g=$ Identity. (The results for the general function $g$ and $t_{e} \neq 0$ can be derived similarly and we state them at the end of the corresponding sections - see (6.25), (7.13) etc.) Then the internal dynamics and the response are given as follows.

$$
\begin{gathered}
\frac{\mathrm{d} y_{2}}{\mathrm{~d} t}=\frac{S(x)-y_{2}}{t_{a}} \\
\lambda(y) \equiv \text { Response }=\lambda_{0}-b\left(S(x)-y_{2}\right)
\end{gathered}
$$

It is convenient to define the new internal state variable $z_{2}$ by the formula

$$
y_{2}=S(x)+z_{2} \quad \Leftrightarrow \quad z_{2}=y_{2}-S(x) \text {. }
$$

Then, we have

$$
\frac{\mathrm{d} z_{2}}{\mathrm{~d} t}=\frac{\mathrm{d}\left(y_{2}-S(x)\right)}{\mathrm{d} t}=\frac{\mathrm{d} y_{2}}{\mathrm{~d} t}-\frac{\mathrm{d} S(x)}{\mathrm{d} t}=\frac{S(x)-y_{2}}{t_{a}}-S^{\prime}(x) \frac{\mathrm{d} x}{\mathrm{~d} t}=-\frac{z_{2}}{t_{a}} \mp S^{\prime}(x) s,
$$

where the sign of the last term is determined by the sign of the velocity of the particle. Moreover,

$$
\lambda\left(z_{2}\right) \equiv \lambda(y)=\lambda_{0}-b\left(S(x)-y_{2}\right)=\lambda_{0}+b z_{2} .
$$

Later we will make use of the following estimate on the internal state, and to avoid repetition, we introduce the following definition. Suppose that the cell moves in 1D according to a velocity jump process with internal dynamics, and that the internal state $z_{2}$ of the cell evolves according to (3.9). We call this the standard process.

LEMMA 3.1. Suppose that the cells execute the standard process, and suppose that

$$
\left|S^{\prime}(x)\right| \leq K, \text { for } x \in \mathbb{R}, \quad{ }_{7} \quad \text { and } \quad\left|z_{2}(0)\right| \leq s t_{a} K
$$


Then, we have

$$
\left|z_{2}(t)\right| \leq s t_{a} K, \text { for } t \geq 0
$$

Proof. If $z_{2}(t)=-s t_{a} K$, then the estimate $\left|S^{\prime}(x)\right| \leq K$ implies $\frac{\mathrm{d} z_{2}}{\mathrm{~d} t} \geq 0$. Similarly, if $z_{2}(t)=s t_{a} K$, then the estimate $\left|S^{\prime}(x)\right| \leq K$ implies $\frac{\mathrm{d} z_{2}}{\mathrm{~d} t} \leq 0$. As $\left|z_{2}(0)\right| \leq s t_{a} K$, we have $\left|z_{2}(t)\right| \leq s t_{a} K$ for all $t \geq 0$.

Q.E.D.

For a physically-reasonable model we must ensure that the turning rate $\lambda\left(z_{2}\right)$ is always nonnegative, and for this we introduce the following standing hypothesis.

$$
\text { Assume that }\left|S^{\prime}(x)\right| \leq \bar{C} \quad \text { where } \bar{C} \text { is given by } \quad \bar{C}=\frac{\lambda_{0}}{b s t_{a}} .
$$

Given (3.11) we have the following lemma.

LEMma 3.2. Suppose that the cells execute the standard process and (3.11) is satisfied. Suppose that initially $\lambda\left(z_{2}(0)\right) \geq 0$. Then, we have

$$
\lambda\left(z_{2}(t)\right) \geq 0 \text { for all } t \geq 0
$$

Proof. The linear turning rate (3.10) is nonnegative if and only if $z_{2} \geq-\lambda_{0} / b$. As $\lambda\left(z_{2}(0)\right) \geq 0$, then we have $z_{2}(0) \geq-\frac{\lambda_{0}}{b}$. Then Lemma 3.1 implies that $z_{2}(t) \geq-\frac{\lambda_{0}}{b}$ for $t \geq 0$. Consequently, the turning rate $\lambda(y)$ is nonnegative for all $t \geq 0$.

Q.E.D.

In view of the preceding assumptions and simplifications, the evolution equations (3.4) - (3.5) for the densities $p^{ \pm}\left(x, z_{2}, t\right)$ can be written as

$$
\begin{aligned}
& \frac{\partial p^{+}}{\partial t}+s \frac{\partial p^{+}}{\partial x}+\frac{\partial}{\partial z_{2}}\left[\left(-\frac{z_{2}}{t_{a}}-s S^{\prime}(x)\right) p^{+}\right]=\left(\lambda_{0}+b z_{2}\right)\left[-p^{+}+p^{-}\right] \\
& \frac{\partial p^{-}}{\partial t}-s \frac{\partial p^{-}}{\partial x}+\frac{\partial}{\partial z_{2}}\left[\left(-\frac{z_{2}}{t_{a}}+s S^{\prime}(x)\right) p^{-}\right]=\left(\lambda_{0}+b z_{2}\right)\left[p^{+}-p^{-}\right] .
\end{aligned}
$$

In the following sections we use equations (3.12) - (3.13) to derive macroscopic equations. First however we address the question of existence and nonnegativity of the densities $p^{ \pm}\left(x, z_{2}, t\right)$. In the following lemma we prove the existence and nonnegativity for the general system (3.4) - (3.5), and this implies the result for (3.12) - (3.13).

Lemma 3.3. Suppose that $f \in C^{1}\left(\mathbb{R}^{m} \times \mathbb{R}^{M}\right)$ and let $S: \mathbb{R} \times[0, \infty) \rightarrow \mathbb{R}^{M}$ be continuous. Moreover, suppose that $\lambda(y)$ in (3.4)-(3.5) is always nonnegative, and that $p_{0}^{+}: \mathbb{R}^{m+1} \rightarrow[0, \infty)$ and $p_{0}^{-}: \mathbb{R}^{m+1} \rightarrow[0, \infty)$ are given nonnegative, compactly-supported $C^{1}$-functions. Then there exists a domain $Q \subset \mathbb{R}^{m+1} \times[0, \infty)$ containing the entire plane $t=0$ such that the system of equations (3.4) - (3.5) with initial conditions $p^{ \pm}(x, y, 0)=p_{0}^{ \pm}(x, y)$ has a unique $C^{1}$-solution in $Q$. Moreover, the $p^{ \pm}$are nonnegative wherever they are defined.

Proof. Our assumptions guarantee the existence of characteristics for both equations in (3.4) - (3.5). Consequently, we can apply the modified implicit function theorem to show a local existence of a unique classical solution (see [3, Section 2.4.4]). To prove nonnegativity, let us consider that the solution is NOT nonnegative and define

$$
t^{0}=\inf \left\{\tau: \text { there exists }(x, y) \text { such that } p^{+}(x, y, \tau)<0 \text { or } p^{-}(x, y, \tau)<0\right\},
$$

i.e., $t^{0}$ is the last time for which the nonnegativity of solutions is satisfied. In particular, we have $p^{ \pm}\left(\cdot, \cdot, t^{0}\right) \geq 0$. 
Let $\chi_{x, y, t^{0}}^{+}(\tau)$ be a characteristic through the point $\left(x, y, t^{0}\right)$ for the equation (3.4), let $\chi_{x, y, t^{0}}^{-}(\tau)$ be a characteristic through the point $\left(x, y, t^{0}\right)$ for the equation (3.5). Thus $\chi_{x, y, t^{0}}^{ \pm}(\tau)$ are curves in the $(m+2)$ dimensional $(x, y, t)$-space along which the equations (3.4) - (3.5) read

$$
\begin{gathered}
\frac{d}{d \tau} p^{+}\left(\chi_{x, y, t^{0}}^{+}(\tau)\right)=-c\left(\chi_{x, y, t^{0}}^{+}(\tau)\right) p^{+}\left(\chi_{x, y, t^{0}}^{+}(\tau)\right)+\lambda\left(\chi_{x, y, t^{0}}^{+}(\tau)\right) p^{-}\left(\chi_{x, y, t^{0}}^{+}(\tau)\right) \\
\frac{d}{d \tau} p^{-}\left(\chi_{x, y, t^{0}}^{-}(\tau)\right)=\lambda\left(\chi_{x, y, t^{0}}^{-}(\tau)\right) p^{+}\left(\chi_{x, y, t^{0}}^{-}(\tau)\right)-c\left(\chi_{x, y, t^{0}}^{-}(\tau)\right) p^{-}\left(\chi_{x, y, t^{0}}^{-}(\tau)\right)
\end{gathered}
$$

wherein

$$
c(x, y, t)=\lambda(y)+\sum_{i=1}^{m} \frac{\partial f_{i}(y, S(x, t))}{\partial y_{i}} .
$$

Let us suppose that $p^{+}\left(x, y, t^{0}\right)=0$. Then (3.14) implies (using $\tau=t^{0}, \lambda(y) \geq 0$ and $p^{-}\left(x, y, t^{0}\right) \geq 0$ )

$$
\frac{d}{d \tau} p^{+}\left(x, y, t^{0}\right) \geq 0
$$

Similarly, if $p^{-}\left(x, y, t^{0}\right)=0$, then (3.15) gives $\frac{d}{d \tau} p^{-}\left(x, y, t^{0}\right) \geq 0$. Consequently, there exists a constant $c>0$ such that the solutions $p^{ \pm}$are nonnegative in the time interval $\left[t^{0}, t^{0}+c\right)$. This is the contradiction with the choice of $t^{0}$.

Q.E.D.

4. Moment equations. The next step is to derive macroscopic equations from the simplified system (3.12) - (3.13). Since there are only two velocities and one internal state variable, the density $n(x, t)$ is given by (compare with (3.3))

$$
n(x, t)=\int_{\mathbb{R}} p^{+}\left(x, z_{2}, t\right)+p^{-}\left(x, z_{2}, t\right) \mathrm{d} z_{2} .
$$

The objective is to derive an evolution equation involving only $n$, if possible. For this purpose define $\mathcal{N}=p^{+}\left(x, z_{2}, t\right)+$ $p^{-}\left(x, z_{2}, t\right)$ and $\mathcal{J}=s\left(p^{+}\left(x, z_{2}, t\right)-p^{-}\left(x, z_{2}, t\right)\right)$; the former is the microscopic particle density, obtained by integrating $p$ over $v$, while the latter is a microscopic flux obtained similarly. In this notation (4.1) can be written

$$
n(x, t)=\int_{\mathbb{R}} \mathcal{N}\left(x, z_{2}, t\right) \mathrm{d} z_{2},
$$

and we define the additional moments

$$
\begin{aligned}
& j(x, t)=\int_{\mathbb{R}} \mathcal{J}\left(x, z_{2}, t\right) \mathrm{d} z_{2}, \\
& n_{1}(x, t)=\int_{\mathbb{R}} z_{2} \mathcal{N}\left(x, z_{2}, t\right) \mathrm{d} z_{2}, \\
& j_{1}(x, t)=\int_{\mathbb{R}} z_{2} \mathcal{J}\left(x, z_{2}, t\right) \mathrm{d} z_{2},
\end{aligned}
$$

and

$$
j_{2}(x, t)=\int_{\mathbb{R}}\left(z_{2}\right)^{2} \mathcal{J}\left(x, z_{2}, t\right) \mathrm{d} z_{2} .
$$

The quantity $j$ is the macroscopic particle flux, $n_{1}$ and $j_{1}$ are first moments with respect to the slow component of the internal state of the microscopic density and flux, respectively, and $j_{2}$ is the second moment of the microscopic flux with respect to the slow component of the internal state. All moments with respect to $z_{2}$ are well-defined by virtue of Lemma 
3.1 and the standing assumption (3.11), which implies that $p$ vanishes identically outside some sufficiently large interval in $\left|z_{2}\right|$.

Next, by multiplying (3.12) and (3.13) by 1 or $z_{2}$, integrating with respect to $z_{2}$, and adding or subtracting the resulting equations, we obtain the following four moment equations.

$$
\begin{aligned}
& \frac{\partial n}{\partial t}+\frac{\partial j}{\partial x}=0 \\
& \frac{\partial j}{\partial t}+s^{2} \frac{\partial n}{\partial x}=-2 \lambda_{0} j-2 b j_{1} \\
& \frac{\partial n_{1}}{\partial t}+\frac{\partial j_{1}}{\partial x}=-S^{\prime}(x) j-\frac{1}{t_{a}} n_{1} \\
& \frac{\partial j_{1}}{\partial t}+s^{2} \frac{\partial n_{1}}{\partial x}=-s^{2} S^{\prime}(x) n-\left(2 \lambda_{0}+\frac{1}{t_{a}}\right) j_{1}-2 b j_{2}
\end{aligned}
$$

We see that the moment equations for a density-flux pair introduces a higher-order flux via the change in turning rate, as measured by $b$. In Section 6 we rescale the variables and then close the system of four moment equations with the assumption that

$$
j_{2}=0
$$

i.e., we simply neglect the second-order flux. The moment closure (4.11) will be rigorously justified in the case of shallow gradients of the signal. The moment closures for arbitrary signal functions will be discussed in Section 9.2.

Of course one can ask what a lower-order closure (i.e. the closure assumption on $j_{1}$ ) leads to, and it is easy to see that if we assume that $j_{1}=0$ we obtain the telegraph equation

$$
\frac{1}{2 \lambda_{0}} \frac{\partial^{2} n}{\partial t^{2}}+\frac{\partial n}{\partial t}=\frac{s^{2}}{2 \lambda_{0}} \frac{\partial^{2} n}{\partial x^{2}}
$$

This equation completely ignores the external signal, and therefore this approximation is not suitable for studying the dependence of $n$ on the signal. Clearly (4.12) applies if there is no effect of the signal on the turning rate, and in this case there can be no taxis.

From (4.7) - (4.10) we can derive evolution equations for various statistics of the motion that give insight into the asymptotics of solutions of the system of moment equations. These are derived in the following section.

5. Evolution of certain statistics of the motion. We denote the total number of the particles in the domain by $n_{0}$, this is given by

$$
n_{0}=\int_{\mathbb{R}} n(x, t) \mathrm{d} x .
$$

The mean position of the particles $\langle x\rangle(t)$ and the mean square displacement $<x^{2}>(t)$ are given by

$$
<x>(t)=\frac{1}{n_{0}} \int_{\mathbb{R}} x n(x, t) \mathrm{d} x, \quad<x^{2}>(t)=\frac{1}{n_{0}} \int_{\mathbb{R}}(x-<x>)^{2} n(x, t) \mathrm{d} x .
$$

We define the spatial moments of $j, j_{1}$, and $j_{2}$ as follows:

$$
j^{0}=\int_{\mathbb{R}} j(x, t) \mathrm{d} x, \quad j^{x}=\int_{\mathbb{R}} x j(x, t) \mathrm{d} x, \quad j_{1}^{0}=\int_{\mathbb{R}} j_{1}(x, t) \mathrm{d} x, \quad j_{2}^{0}=\int_{\mathbb{R}} j_{2}(x, t) \mathrm{d} x .
$$

Then, multiplying (4.7) by $x$ and by $(x-<x>(t))^{2}$, respectively, and integrating the resulting equations with respect to $x$, we find that

$$
\frac{\mathrm{d}}{\mathrm{d} t}<x>=\frac{j^{0}}{n_{0}} \quad \text { and } \quad \frac{\mathrm{d}}{\mathrm{d} t}<x^{2}>=\frac{2 j^{x}-2<x>j^{0}}{n_{0}}
$$


Integrating (4.8) and (4.10) with respect to $x$, we obtain the evolution equations for $j^{0}$ and $j_{1}^{0}$ :

$$
\begin{gathered}
\frac{\mathrm{d}}{\mathrm{d} t} j^{0}=-2 \lambda_{0} j^{0}-2 b j_{1}^{0}, \\
\frac{\mathrm{d}}{\mathrm{d} t} j_{1}^{0}=-s^{2} \int_{\mathbb{R}} S^{\prime}(x) n(x, t) \mathrm{d} x-\left(2 \lambda_{0}+\frac{1}{t_{a}}\right) j_{1}^{0}-2 b j_{2}^{0} .
\end{gathered}
$$

We can solve the system of equations (5.3) - (5.4) explicitly for $j^{0}$ as a function of the quantities $\int_{\mathbb{R}} S^{\prime}(x) n(x, t) \mathrm{d} x$ and $j_{2}^{0}$. Consequently, using (5.2), we obtain the evolution equation for $\langle x\rangle$ in the following form

$$
\frac{\mathrm{d}}{\mathrm{d} t}<x>=\frac{e^{-2 \lambda_{0} t}}{n_{0}}\left[j^{0}(0)+\int_{0}^{t} e^{t^{\prime} / t_{a}} 2 b\left(-j_{1}^{0}(0)+\int_{0}^{t^{\prime}} e^{2 \lambda_{0} t^{\prime \prime}+t^{\prime \prime} / t_{a}}\left\{s^{2} \int_{\mathbb{R}} S^{\prime}(x) n(x, t) \mathrm{d} x+2 b j_{2}^{0}\right\} \mathrm{d} t^{\prime \prime}\right) \mathrm{d} t^{\prime}\right] .
$$

Thus the mean displacement is driven by the flux $j^{0}$, which is in turn forced by the projection of the local density onto the gradient, as given by the integral term in $S^{\prime}$, as well as by the higher-order flux $j_{2}^{0}$. From the foregoing one can conclude that if $\langle x\rangle$ tends to a constant as $t \rightarrow \infty$, then the total flux $j^{0}$ must vanish as $t \rightarrow \infty$, and this in turn requires that the term $s^{2} \int_{\mathbb{R}} S^{\prime}(x) n(x, t) \mathrm{d} x+2 b j_{2}^{0}$ must tend to zero. Thus this is a necessary, but not sufficient, condition for steady patterns. Similarly, one can derive the system of evolution equations for the mean square displacement.

In order to gain some further insights into the evolution of the statistics of motion, let us suppose that

$$
S^{\prime}(x)=C=\text { constant } \quad \text { and } \quad j_{2}=0 .
$$

To derive equations for the mean position $\langle x\rangle$ and the mean square displacement $\left\langle x^{2}\right\rangle$ under this restriction we introduce some additional moments

$$
n_{1}^{0}=\int_{\mathbb{R}} n_{1}(x, t) \mathrm{d} x, \quad n_{1}^{x}=\int_{\mathbb{R}} x n_{1}(x, t) \mathrm{d} x, \quad \text { and } \quad j_{1}^{x}=\int_{\mathbb{R}} x j_{1}(x, t) \mathrm{d} x .
$$

Then, integrating (4.8), (4.9) and (4.10) with respect to $x$, we obtain the system

$$
\frac{\mathrm{d}}{\mathrm{d} t}\left(\begin{array}{c}
j^{0} \\
n_{1}^{0} \\
j_{1}^{0}
\end{array}\right)=\left(\begin{array}{ccc}
-2 \lambda_{0} & 0 & -2 b \\
-C & -\frac{1}{t_{a}} & 0 \\
0 & 0 & -\left(2 \lambda_{0}+\frac{1}{t_{a}}\right)
\end{array}\right)\left(\begin{array}{c}
j^{0} \\
n_{1}^{0} \\
j_{1}^{0}
\end{array}\right)+\left(\begin{array}{c}
0 \\
0 \\
-s^{2} C n_{0}
\end{array}\right) .
$$

Similarly, multiplying (4.8), (4.9) and (4.10) by $x$ and integrating with respect to $x$, we obtain

$$
\frac{\mathrm{d}}{\mathrm{d} t}\left(\begin{array}{c}
j^{x} \\
n_{1}^{x} \\
j_{1}^{x}
\end{array}\right)=\left(\begin{array}{ccc}
-2 \lambda_{0} & 0 & -2 b \\
-C & -\frac{1}{t_{a}} & 0 \\
0 & 0 & -\left(2 \lambda_{0}+\frac{1}{t_{a}}\right)
\end{array}\right)\left(\begin{array}{c}
j^{x} \\
n_{1}^{x} \\
j_{1}^{x}
\end{array}\right)+\left(\begin{array}{c}
s^{2} n_{0} \\
j_{1}^{0} \\
s^{2} n_{1}^{0}-s^{2} C<x>n_{0}
\end{array}\right) .
$$

Together (5.5) and (5.6) form a system of six linear nonhomogeneous equations. The linear part of this system has all eigenvalues strictly negative, and since $n_{0}$ is constant it follows that the system has a unique stable steady state given by

$$
j^{0}=\frac{b s^{2} C t_{a}}{\lambda_{0}+2 \lambda_{0}^{2} t_{a}} n_{0}, \quad \text { and } \quad 2 j^{x}-2<x>j^{0}=\left(\frac{s^{2}}{\lambda_{0}}+\frac{2 b^{2} s^{4} C^{2} t_{a}^{3}}{\left(\lambda_{0}+2 \lambda_{0}^{2} t_{a}\right)^{2}}\right) n_{0} .
$$

Thus, (5.2) implies that asymptotically for $t \rightarrow \infty$, we have

$$
<x>(t)=\frac{b s^{2} C t_{a}}{\lambda_{0}+2 \lambda_{0}^{2} t_{a}} t, \quad \text { and } \quad<x^{2}>(t)=\left(\frac{s^{2}}{\lambda_{0}}+\frac{2 b^{2} s^{4} C^{2} t_{a}^{3}}{\left(\lambda_{0}+2 \lambda_{0}^{2} t_{a}\right)^{2}}\right) t .
$$

The second of these shows that when the gradient $S^{\prime}(x)=C$ the standard process is asymptotically a diffusion process with diffusion constant

$$
D=\frac{s^{2}}{2 \lambda_{0}}+\frac{b^{2} s^{4} C^{2} t_{a}^{3}}{\left(\lambda_{0}+2 \lambda_{0}^{2} t_{a}\right)^{2}} .
$$

Later, using scaling (6.7), we will see that the second term in (5.8) is smaller than the first and then the equation (5.8) gives $D \sim \frac{s^{2}}{2 \lambda_{0}}$. 
6. Hyperbolic scaling - derivation of modified classical chemotaxis equation. The macroscopic equations that can be obtained from the moment equations depend on the time and space scales of interest. In this section we use a hyperbolic scaling of space and time that is valid for short times, which leads to a hyperbolic version of the classical chemotaxis equation. In Section 7, we use parabolic scaling valid for large times, which leads to the classical chemotaxis equation.

Let $L$, and $T$ be scale factors for the length, and time, respectively, let $N_{0}$ be a scale factor for the particle density, and define the dimensionless variables

$$
\hat{x}=\frac{x}{L} \quad \hat{t}=\frac{t}{T} \quad \hat{n}=\frac{n}{N_{0}} \quad \hat{j}=\frac{j T}{N_{0} L} \quad \hat{n}_{1}=\frac{n_{1}}{N_{0}} \quad \hat{j}_{1}=\frac{j_{1} T}{N_{0} L} \quad \text { and } \quad \hat{j}_{2}=\frac{j_{2} T}{N_{0} L} .
$$

Then the moment equations can be written in the dimensionless form

$$
\begin{aligned}
& \frac{\partial \hat{n}}{\partial \hat{t}}+\frac{\partial \hat{j}}{\partial \hat{x}}=0 \\
& \frac{\partial \hat{j}}{\partial \hat{t}}+\hat{s}^{2} \frac{\partial \hat{n}}{\partial \hat{x}}=-2 \hat{\lambda}_{0} \hat{j}-2 \hat{b} \hat{j}_{1} \\
& \frac{\partial \hat{n}_{1}}{\partial \hat{t}}+\frac{\partial \hat{j}_{1}}{\partial \hat{x}}=-\hat{S}^{\prime}(\hat{x}) \hat{j}-\frac{1}{\hat{t}_{a}} \hat{n}_{1} \\
& \frac{\partial \hat{j}_{1}}{\partial \hat{t}}+\hat{s}^{2} \frac{\partial \hat{n}_{1}}{\partial \hat{x}}=-\hat{s}^{2} \hat{S}^{\prime}(\hat{x}) \hat{n}-\left(2 \hat{\lambda}_{0}+\frac{1}{\hat{t}_{a}}\right) \hat{j}_{1}-2 \hat{b} \hat{j}_{2}
\end{aligned}
$$

where

$$
\hat{s} \equiv\left(\frac{s_{0} T}{L}\right) \quad \hat{\lambda}_{0} \equiv \lambda_{0} T \quad \hat{b} \equiv b T \quad \text { and } \quad \hat{t}_{a} \equiv \frac{t_{a}}{T}
$$

In order to derive the macroscopic equations, we have to specify $L$ and $T$ and estimate the dimensionless parameters. Here, we choose

$$
T=1 \mathrm{sec} \quad \text { and } L=1 \mathrm{~mm}
$$

i.e., we use a time scale that is of the same order as the mean time between directional changes. A typical speed of a bacterium is $s=10-20 \mu / \mathrm{sec}$, and assuming that the adaptation time and the bias are also of the same order as the mean run time, we get

$$
\hat{s} \sim \mathcal{O}(\varepsilon), \quad \hat{\lambda}_{0} \sim \hat{b} \sim \hat{t}_{a} \sim \mathcal{O}(1), \quad \text { where } \varepsilon \approx 10^{-2} .
$$

The scaling (6.6) - (6.7) will lead to a hyperbolic version of a classical chemotaxis equation. For simplicity, we drop the hats on $x, t, s, \lambda_{0}, b, t_{a}, S$ and the hats on moments, and use the same symbols for the dimensionless variables. Moreover, as $\hat{s} \equiv s$ is the small parameter and all other parameters are $\mathcal{O}(1)$, we set $s=\varepsilon$ to emphasize the smallness of the scaled velocity $s$.

Then the moment equations read as follows:

$$
\begin{aligned}
& \frac{\partial n}{\partial t}+\frac{\partial j}{\partial x}=0 \\
& \frac{\partial j}{\partial t}+\varepsilon^{2} \frac{\partial n}{\partial x}=-2 \lambda_{0} j-2 b j_{1} \\
& \frac{\partial n_{1}}{\partial t}+\frac{\partial j_{1}}{\partial x}=-S^{\prime}(x) j-\frac{1}{t_{a}} n_{1} \\
& \frac{\partial j_{1}}{\partial t}+\varepsilon^{2} \frac{\partial n_{1}}{\partial x}=-\varepsilon^{2} S^{\prime}(x) n-\left(2 \lambda_{0}+\frac{1}{t_{a}}\right) j_{1}-2 b j_{2} .
\end{aligned}
$$


In order to close this system we have to specify

$$
j_{2}=\mathcal{F}\left(n, j, n_{1}, j_{1}\right)
$$

where the functional $\mathcal{F}$ is to be determined. To do that, we first rewrite our standing assumption (3.11) using hyperbolic scaling (6.6) - (6.7). We denote the dimensionless constant $\bar{C}$ again as $\bar{C}$ for simplicity, and then (3.11) for the dimensionless signal gradient reads as follows.

$$
S^{\prime}(x) \in[-\bar{C}, \bar{C}] \quad \text { where } \quad \bar{C} \sim \mathcal{O}\left(\frac{1}{\varepsilon}\right)
$$

Thus the maximal possible gradient that satisfies (3.11) is $\mathcal{O}(1 / \varepsilon)$ on the hyperbolic scale. In other words, the restriction on the gradients that guarantees positivity of the turning rate is very weak, and we strengthen it as follows.

DEFINITION 6.1. We call the signal gradient shallow on the hyperbolic scale if

$$
S^{\prime}(x) \in[-\bar{K}, \bar{K}] \quad \text { where } \quad \bar{K} \sim \mathcal{O}(1) .
$$

In the following, we investigate the case of shallow gradients, and to do that, we have to estimate the moments in (6.8) (6.11).

Lemma 6.2. Suppose that the signal gradient is shallow. Then the moments in (6.8)-(6.11) can be estimated as follows.

$$
\frac{j}{n} \leq \varepsilon K_{1}, \quad \frac{n_{1}}{n} \leq \varepsilon K_{2}, \quad \frac{j_{1}}{n} \leq \varepsilon^{2} K_{3}, \quad \frac{j_{2}}{n} \leq \varepsilon^{3} K_{4},
$$

where the constants $K_{1}, K_{2}, K_{3}$, and $K_{4}$ are $\mathcal{O}(1)$.

Proof. We use (4.6), nonnegativity of $p^{ \pm}$, Lemma 3.1 and (4.1) to estimate

$$
\begin{gathered}
j_{2}=\varepsilon \int_{\mathbb{R}}\left(z_{2}\right)^{2}\left[p^{+}\left(x, z_{2}, t\right)-p^{+}\left(x, z_{2}, t\right)\right] \mathrm{d} z_{2} \leq \varepsilon \int_{\mathbb{R}}\left(z_{2}\right)^{2}\left[p^{+}\left(x, z_{2}, t\right)+p^{+}\left(x, z_{2}, t\right)\right] \mathrm{d} z_{2} \leq \\
\leq \varepsilon^{3}\left(\bar{K} t_{a}\right)^{2} \int_{\mathbb{R}}\left[p^{+}\left(x, z_{2}, t\right)+p^{+}\left(x, z_{2}, t\right)\right] \mathrm{d} z_{2}=\varepsilon^{3}\left(\bar{K} t_{a}\right)^{2} n=\varepsilon^{3} K_{4} n
\end{gathered}
$$

where $K_{4} \sim \mathcal{O}(1)$. This proves the last inequality in (6.15), and the proof of the other inequalities is similar.

Q.E.D.

Therefore the term $2 b j_{2}$ in equation (6.11) is $\mathcal{O}\left(\varepsilon^{3}\right)$ when the gradient is shallow, and we can close the moment equations $(6.8)-(6.11)$ with the moment closure assumption

$$
j_{2}=0 .
$$

This will introduce the error of order $\mathcal{O}\left(\varepsilon^{3}\right)$ into the equation (6.11). Using Lemma 6.2 again, we see that the remaining terms in the equation (6.11) are of order $\mathcal{O}\left(\varepsilon^{2}\right)$ except of the term $\varepsilon^{2} \partial n_{1} / \partial x$, which is of order $\mathcal{O}\left(\varepsilon^{3}\right)$. Hence, we can also neglect the term $\varepsilon^{2} \partial n_{1} / \partial x$ in equation (6.11). Then the moment equations (6.8), (6.9) and (6.11) read as follows.

$$
\begin{gathered}
\frac{\partial n}{\partial t}+\frac{\partial j}{\partial x}=0 \\
\frac{\partial j}{\partial t}+\varepsilon^{2} \frac{\partial n}{\partial x}=-2 \lambda_{0} j-2 b j_{1}, \\
\frac{\partial j_{1}}{\partial t}=-\varepsilon^{2} S^{\prime}(x) n-\left(2 \lambda_{0}+\frac{1}{t_{a}}\right) j_{1}+\mathcal{O}\left(\varepsilon^{3}\right) .
\end{gathered}
$$


The last equation (6.19) can be solved by the method of variation of parameters to obtain

$$
j_{1}(x, t)=\exp \left[-\left(2 \lambda_{0}+\frac{1}{t_{a}}\right) t\right] j_{1}(x, 0)-\varepsilon^{2} S^{\prime}(x) \int_{0}^{t} \exp \left[\left(2 \lambda_{0}+\frac{1}{t_{a}}\right)(s-t)\right] n(x, s) \mathrm{d} s+\mathcal{O}\left(\varepsilon^{3}\right) .
$$

Using the assumption that initially all individuals were perfectly adapted, i.e., $j_{1}(x, 0)=0$, and using integration by parts, we have

$$
\begin{gathered}
j_{1}(x, t)=-\varepsilon^{2} S^{\prime}(x) \int_{0}^{t} \exp \left[\left(2 \lambda_{0}+\frac{1}{t_{a}}\right)(s-t)\right] n(x, s) \mathrm{d} s+\mathcal{O}\left(\varepsilon^{3}\right)= \\
=-\frac{\varepsilon^{2} S^{\prime}(x) t_{a}}{1+2 \lambda_{0} t_{a}}\left\{1-\exp \left[-\left(2 \lambda_{0}+\frac{1}{t_{a}}\right) t\right]\right\} n(x, t)+ \\
+\varepsilon^{2} S^{\prime}(x) \frac{t_{a}}{1+2 \lambda_{0} t_{a}} \int_{0}^{t} \exp \left[\left(2 \lambda_{0}+\frac{1}{t_{a}}\right)(s-t)\right] \frac{\partial n}{\partial t}(x, s) \mathrm{d} s+\mathcal{O}\left(\varepsilon^{3}\right) .
\end{gathered}
$$

Using equation (6.17) and Lemma 6.2, we see that the second term is $\mathrm{O}\left(\varepsilon^{3}\right)$. Neglecting this term and the exponential decaying part of the first term, we obtain the following approximation

$$
j_{1}(x, t)=-\frac{\varepsilon^{2} S^{\prime}(x) t_{a}}{1+2 \lambda_{0} t_{a}} n(x, t)+\mathcal{O}\left(\varepsilon^{3}\right) .
$$

This can be used to reduce the system (6.17) - (6.19) to the following system of two equations

$$
\begin{gathered}
\frac{\partial n}{\partial t}+\frac{\partial j}{\partial x}=0 \\
\frac{\partial j}{\partial t}+\varepsilon^{2} \frac{\partial n}{\partial x}=-2 \lambda_{0} j+2 b \frac{\varepsilon^{2} S^{\prime}(x) t_{a}}{1+2 \lambda_{0} t_{a}} n+\mathcal{O}\left(\varepsilon^{3}\right) .
\end{gathered}
$$

The last step is to reduce these two equations to one equation for $n$. To this end, we differentiate equation (6.21) with respect to $t$ and equation (6.22) with respect to $x$ to obtain

$$
\frac{\partial^{2} n}{\partial t^{2}}+\frac{\partial^{2} j}{\partial t \partial x}=0, \quad \frac{\partial^{2} j}{\partial x \partial t}+\varepsilon^{2} \frac{\partial^{2} n}{\partial x^{2}}=-2 \lambda_{0} \frac{\partial j}{\partial x}+2 b \frac{\partial}{\partial x} \frac{\varepsilon^{2} S^{\prime}(x) t_{a}}{1+2 \lambda_{0} t_{a}} n+\mathcal{O}\left(\varepsilon^{3}\right) .
$$

Hence, solving for $n$, we obtain

$$
\frac{\partial^{2} n}{\partial t^{2}}+2 \lambda_{0} \frac{\partial n}{\partial t}=\frac{\partial}{\partial x}\left(\varepsilon^{2} \frac{\partial n}{\partial x}-\frac{2 b \varepsilon^{2} t_{a}}{1+2 \lambda_{0} t_{a}} S^{\prime}(x) n\right)+\mathcal{O}\left(\varepsilon^{3}\right) .
$$

Recall that we used the notation $s=\varepsilon$ to emphasize the smallness of the scaled speed $s$, and if we revert to using $s$ and drop the $\mathcal{O}\left(\varepsilon^{3}\right)$ error in (6.23), we obtain the hyperbolic version of the classical chemotaxis equation (compare with (1.5)).

$$
\frac{\partial^{2} n}{\partial t^{2}}+2 \lambda_{0} \frac{\partial n}{\partial t}=\frac{\partial}{\partial x}\left(s^{2} \frac{\partial n}{\partial x}-\frac{2 b s^{2} t_{a}}{1+2 \lambda_{0} t_{a}} S^{\prime}(x) n\right) .
$$

Here, the chemotactic sensitivity is given by

$$
\chi=\frac{b s^{2} t_{a}}{\lambda_{0}+2 \lambda_{0}^{2} t_{a}}
$$

which we have already derived for the case in which the signal gradient is constant (see (5.7)). Equation (6.24) was derived for the simplified cartoon model (3.6), but a similar analysis can be done for the full model (2.2) - (2.3) with $t_{e} \neq 0$ and a general function $g$. This leads to the following modified classical chemotaxis equation (cf. (6.24))

$$
\frac{\partial^{2} n}{\partial t^{2}}+2 \lambda_{0} \frac{\partial n}{\partial t}=\frac{\partial}{\partial x}\left(s^{2} \frac{\partial n}{\partial x}-g^{\prime}(S(x)) \frac{2 b s^{2} t_{a}}{\left(1+2 \lambda_{0} t_{a}\right)\left(1+2 \lambda_{0} t_{e}\right)} S^{\prime}(x) m\right)
$$


Now the chemotactic sensitivity is given by

$$
\chi=g^{\prime}(S(x)) \frac{b s^{2} t_{a}}{\lambda_{0}\left(1+2 \lambda_{0} t_{a}\right)\left(1+2 \lambda_{0} t_{e}\right)} .
$$

Note that we can derive (6.24) as the limit $t_{e} \rightarrow 0$ of the equation (6.25) for $g=$ Identity. It should also be noted that in either case (and those that follow) the chemotactic sensitivity vanishes as $t_{a} \rightarrow 0$, which is to be expected since the system adapts instantaneously in this case. One cannot extract the effect of the opposite limit $t_{a} \rightarrow \infty$ as directly, since the scaling assumption that leads to (6.26) has $t_{a} \sim \mathcal{O}(1)$.

6.1. An alternate route to the chemotaxis equation. The previous derivation of (6.24) was done by estimating carefully all terms in the system (6.8) - (6.11) using Lemma 6.2 to estimate the size of moments. That lemma shows that the moments are small compared to the total density of particles $n$. This size order stems from the conditions that (a) the scaled speed $s$ is small, and (b) the internal variable $z_{2}$ is small. The first condition is always true under the scaling (6.6) (6.7), but the internal variable $z_{2}$ is small only under the influence of shallow signal gradients.

Therefore an alternate way to analyze the system $(6.8)-(6.11)$ is to first rescale the fluxes. Define

$$
\bar{j}=\frac{j}{s}, \quad \overline{j_{1}}=\frac{j_{1}}{s} .
$$

Then $j, j_{1}$ are of the same order as corresponding densities $n, n_{1}$ and we can write the system (6.8) - (6.11) in the following matrix form

$$
\frac{\partial v}{\partial t}+s \frac{\partial}{\partial x}(A v)=B(x) v+r
$$

where

$$
v=\left(\begin{array}{c}
n \\
\bar{j} \\
n_{1} \\
\bar{j}_{1}
\end{array}\right), \quad A=\left(\begin{array}{llll}
0 & 1 & 0 & 0 \\
1 & 0 & 0 & 0 \\
0 & 0 & 0 & 1 \\
0 & 0 & 1 & 0
\end{array}\right), \quad r=\left(\begin{array}{c}
0 \\
0 \\
0 \\
-2 b j_{2}
\end{array}\right) \text {, }
$$

and

$$
B(x)=\left(\begin{array}{cccc}
0 & 0 & 0 & 0 \\
0 & -2 \lambda_{0} & 0 & -2 b \\
0 & -s S^{\prime}(x) & -\frac{1}{t_{a}} & 0 \\
-s S^{\prime}(x) & 0 & 0 & -\left(2 \lambda_{0}+\frac{1}{t_{a}}\right)
\end{array}\right) .
$$

Equation (6.28) holds for any signal function satisfying the standing assumption (3.11), since it is just a different formulation of the system (6.8) - (6.11) obtained by rescaling of fluxes (6.27). Therefore, using Lemma 6.2, we could derive the equation (6.24) as before. However, once we use the moment closure $j_{2}=0$, we do not need to estimate internal moments to derive (6.24), and we can derive the equation (6.24) using the following heuristic.

Assuming $j_{2}=0$, the system (6.28) can be written in the form

$$
\frac{\partial v}{\partial t}+s \frac{\partial}{\partial x}(A v)=B(x) v .
$$

This is a hyperbolic system of four linear PDEs with nonconstant coefficients for four unknowns $n, j, n_{1}$, and $j_{1}$. The matrix $B(x)$ has the interesting property that its eigenvalues do not depend on the signal $S(x)$, and consequently, the eigenvalues of $B(x)$ are independent of $x$. An easy calculation gives the following four (not necessarily distinct) eigenvalues of $B(x)$ :

$$
\lambda_{1}=0, \quad \lambda_{2}=-2 \lambda_{0}, \quad \lambda_{3}=-\frac{1}{t_{a}}, \quad \lambda_{4}=-\frac{1+2 \lambda_{0} t_{a}}{t_{a}} .
$$

Let us note that three of the eigenvalues $\lambda_{2}, \lambda_{3}$ and $\lambda_{4}$ are negative. Moreover, $\lambda_{4}<\lambda_{2}$ and $\lambda_{4}<\lambda_{3}$. 
System (6.31) cannot be solved explicitly, so we simplify it as follows. First consider the system (6.31) with $s=0$, in which case (6.31) reduces to the system of ordinary differential equations

$$
\frac{\partial w}{\partial t}=B(x) w
$$

where the matrix $B(x)$ has four eigenvalues given by (6.32). Consequently, the long time behavior is given by the eigenvectors corresponding to the largest eigenvalues. As $s$ is a small parameter, we use the following heuristic argument to derive the modified classical chemotaxis equation.

The eigenvectors of $B(x)$ are:

$$
\begin{aligned}
& \lambda_{1}=0: \quad \vartheta_{1}=\left(\begin{array}{c}
\lambda_{0}+2 \lambda_{0}^{2} t_{a} \\
b s S^{\prime}(x) t_{a} \\
-b\left(s S^{\prime}(x) t_{a}\right)^{2} \\
-s S^{\prime}(x) t_{a} \lambda_{0}
\end{array}\right), \quad \lambda_{2}=-2 \lambda_{0}: \quad \vartheta_{2}=\left(\begin{array}{c}
0 \\
-1+2 \lambda_{0} t_{a} \\
s S^{\prime}(x) t_{a} \\
0
\end{array}\right) \text {, } \\
& \lambda_{3}=-\frac{1}{t_{a}}: \quad \vartheta_{3}=\left(\begin{array}{l}
0 \\
0 \\
1 \\
0
\end{array}\right), \quad \lambda_{4}=-\frac{1+2 \lambda_{0} t_{a}}{t_{a}}: \quad \vartheta_{4}=\left(\begin{array}{c}
0 \\
2 b \lambda_{0} t_{a} \\
s S^{\prime}(x) b t_{a} \\
\lambda_{0}
\end{array}\right) .
\end{aligned}
$$

Let us suppose that $2 \lambda_{0} \neq \frac{1}{t_{a}}$. Then, we can write the unknown vector function $v(x, t)$ as a linear combination of the eigenvectors $\vartheta_{i}, i=1, \ldots, 4$, i.e.

$$
v(x, t)=c_{1}(x, t) \vartheta_{1}+c_{2}(x, t) \vartheta_{2}+c_{3}(x, t) \vartheta_{3}+c_{4}(x, t) \vartheta_{4} .
$$

We are interested in the evolution of the first component of $v$ which is $n$. The first component is nonzero only for the vector $\vartheta_{1}$, and consequently we have

$$
n(x, t)=\left(\lambda_{0}+2 \lambda_{0}^{2} t_{a}\right) c_{1}(x, t) .
$$

Then (6.35) reads as follows

$$
v(x, t)=\frac{n(x, t)}{\left(\lambda_{0}+2 \lambda_{0}^{2} t_{a}\right)} \vartheta_{1}+c_{2}(x, t) \vartheta_{2}+c_{3}(x, t) \vartheta_{3}+c_{4}(x, t) \vartheta_{4} .
$$

The scaled velocity $s$ is small comparable to $2 \lambda_{0}+\frac{1}{t_{a}}$ (see (6.7)). Consequently, the major dynamical features will be given by the eigenvectors corresponding to the zero eigenvalue and the eigenvalues with lower absolute value. We have the inequalities

$$
\lambda_{4}<\lambda_{2}<\lambda_{1}=0, \quad \text { and } \quad \lambda_{4}<\lambda_{3}<\lambda_{1}=0 \text {, }
$$

and therefore we consider the projection

$$
v(x, t)=\frac{n(x, t)}{\left(\lambda_{0}+2 \lambda_{0}^{2} t_{a}\right)} \vartheta_{1}+c_{2}(x, t) \vartheta_{2}+c_{3}(x, t) \vartheta_{3},
$$

to obtain (from the fourth component of the vector $v$ )

$$
\overline{j_{1}}(x, t)=\frac{-s S^{\prime}(x) t_{a}}{\left(1+2 \lambda_{0} t_{a}\right)} n(x, t) .
$$

But this is precisely the formula (6.20), and hence we can continue as before to derive the modified classical chemotaxis equation (6.24). 
7. Parabolic scaling - derivation of the classical chemotaxis equation. The previous analysis used the scaling (6.6) - (6.7) and we derived the modified classical chemotaxis equation (6.24). This equation formally reduces for large times to the classical chemotaxis equation (1.5). However, the equation (6.24) was derived on a small time scale, so to make the connection between (6.24) and (1.5) more rigorous, we introduce the parabolic scaling which leads directly to the classical chemotaxis equation. To avoid confusion, let us note that in the parabolic scaling all microscopic parameters (including the scaled velocity $s$ ) in the resulting equations will be $\mathcal{O}(1)$ and the scaling parameter $\varepsilon$ does NOT have the meaning of the scaled velocity as it did in the previous Section 6.

Following [18], we introduce the scaling parameter $\varepsilon$ and we use the parabolic scales of space and time given by

$$
T=\frac{1}{\varepsilon^{2}} T_{1}, \quad L=\frac{1}{\varepsilon} \frac{s_{0}}{L_{1} / T_{1}} L_{1},
$$

where $T_{1}=1 \mathrm{sec}, L_{1}=1 \mathrm{~mm}$ are the scales for space and time used in the hyperbolic scaling, and $s_{0}=10 \mu / \mathrm{sec}$ is a scale factor for velocity. Let $N_{0}$ be a scale factor for the particle density, and define the dimensionless variables

$$
\hat{x}=\frac{x}{L} \quad \hat{t}=\frac{t}{T} \quad \hat{s}=\frac{s}{s_{0}} \quad \hat{n}=\frac{n}{N_{0}} \quad \hat{j}=\frac{j}{N_{0} s_{0}} \quad \hat{n}_{1}=\frac{n_{1}}{N_{0}} \quad \hat{j}_{1}=\frac{j_{1}}{N_{0} s_{0}} \quad \text { and } \quad \hat{j}_{2}=\frac{j_{2}}{N_{0} s_{0}} .
$$

Next, let us denote

$$
\hat{s}=\frac{s}{s_{0}} \quad \hat{\lambda}_{0} \equiv \lambda_{0} T_{1} \quad \hat{b} \equiv b T_{1} \quad \text { and } \quad \hat{t}_{a} \equiv \frac{t_{a}}{T_{1}} .
$$

Then, on the parabolic time scale, we have

$$
\hat{s} \sim \hat{\lambda}_{0} \sim \hat{b} \sim \hat{t}_{a} \sim \mathcal{O}(1)
$$

and the dimensionless equations take the form

$$
\begin{aligned}
& \varepsilon^{2} \frac{\partial \hat{n}}{\partial \hat{t}}+\varepsilon \frac{\partial \hat{j}}{\partial \hat{x}}=0 \\
& \varepsilon^{2} \frac{\partial \hat{j}}{\partial \hat{t}}+\varepsilon \hat{s}^{2} \frac{\partial \hat{n}}{\partial \hat{x}}=-2 \hat{\lambda}_{0} \hat{j}-2 \hat{b} \hat{j}_{1} \\
& \varepsilon^{2} \frac{\partial \hat{n}_{1}}{\partial \hat{t}}+\varepsilon \frac{\partial \hat{j}_{1}}{\partial \hat{x}}=-\varepsilon \hat{S}^{\prime}(\hat{x}) \hat{j}-\frac{1}{\hat{t}_{a}} \hat{n}_{1} \\
& \varepsilon^{2} \frac{\partial \hat{j}_{1}}{\partial \hat{t}}+\varepsilon \hat{s}^{2} \frac{\partial \hat{n}_{1}}{\partial \hat{x}}=-\varepsilon \hat{s}^{2} \hat{S}^{\prime}(\hat{x}) \hat{n}-\left(2 \hat{\lambda}_{0}+\frac{1}{\hat{t}_{a}}\right) \hat{j}_{1}-2 \hat{b} \hat{j}_{2}
\end{aligned}
$$

For simplicity, we drop the hats in (7.3) - (7.6), and we consider the case of shallow gradients $S^{\prime}(x) \sim \mathcal{O}(1)$ as before. So, we can use the moment closure $j_{2}=0$ as before, and (7.3) - (7.6) can be written in the following matrix form

$$
\varepsilon^{2} \frac{\partial v}{\partial t}+\varepsilon \frac{\partial}{\partial x}(P v)=\varepsilon Q(x) v+R v
$$

where

$$
\begin{gathered}
v=\left(\begin{array}{c}
n \\
j \\
n_{1} \\
j_{1}
\end{array}\right), \quad P=\left(\begin{array}{cccc}
0 & 1 & 0 & 0 \\
s^{2} & 0 & 0 & 0 \\
0 & 0 & 0 & 1 \\
0 & 0 & s^{2} & 0
\end{array}\right), \\
Q(x)=\left(\begin{array}{cccc}
0 & 0 & 0 & 0 \\
0 & 0 & 0 & 0 \\
0 & -S^{\prime}(x) & 0 & 0 \\
-s^{2} S^{\prime}(x) & 0 & 0 & 0
\end{array}\right), \quad \text { and } \quad R=\left(\begin{array}{cccc}
0 & 0 & 0 & 0 \\
0 & -2 \lambda_{0} & 0 & -2 b \\
0 & 0 & -\frac{1}{t_{a}} & 0 \\
0 & 0 & 0 & -\left(2 \lambda_{0}+\frac{1}{t_{a}}\right)
\end{array}\right) .
\end{gathered}
$$


Here all the entries of the matrices $P, Q(x)$ and $R$ are $\mathcal{O}(1)$. Assuming the regular perturbation expansion

$$
v=v^{0}+\varepsilon v^{1}+\varepsilon^{2} v^{2}+\cdots, \quad \text { where } \quad v^{0}=\left(\begin{array}{c}
n^{0} \\
j^{0} \\
n_{1}^{0} \\
j_{1}^{0}
\end{array}\right), \quad \text { and } \quad v^{1}=\left(\begin{array}{c}
n^{1} \\
j^{1} \\
n_{1}^{1} \\
j_{1}
\end{array}\right)
$$

substituting this into equation (7.7) and comparing terms of equal order in $\varepsilon$, we obtain

$$
\begin{array}{ll}
\varepsilon^{0}: & R v^{0}=0 \\
\varepsilon^{1}: & \frac{\partial}{\partial x}\left(P v^{0}\right)-Q(x) v^{0}=R v^{1} \\
\varepsilon^{2}: & \frac{\partial v^{0}}{\partial t}+\frac{\partial}{\partial x}\left(P v^{1}\right)-Q(x) v^{1}=R v^{2} .
\end{array}
$$

The first equation (7.8) implies that

$$
v_{0}=\left(n^{0}, 0,0,0\right)^{T},
$$

consequently, the second equation (7.9) implies

$$
j^{1}=-\frac{s^{2}}{2 \lambda_{0}} \frac{\partial n^{0}}{\partial x}+\frac{b t_{a} s^{2}}{\lambda_{0}+2 \lambda_{0}^{2} t_{a}} S^{\prime}(x) n^{0} .
$$

Finally, equation (7.10) implies that the left hand side

$$
\frac{\partial v^{0}}{\partial t}+\frac{\partial}{\partial x}\left(P v^{1}\right)-Q(x) v^{1}
$$

is in the range of the operator $\mathbb{R}: w \rightarrow R w$. Consequently, using a Fredholm alternative, the left hand side must be orthogonal to the vector $(1,0,0,0)^{T}$. Hence,

$$
\frac{\partial n^{0}}{\partial t}+\frac{\partial j^{1}}{\partial x}=0
$$

Finally, using (7.11), we derive the classical chemotaxis equation in the following form

$$
\frac{\partial n^{0}}{\partial t}=\frac{\partial}{\partial x}\left(\frac{s^{2}}{2 \lambda_{0}} \frac{\partial n^{0}}{\partial x}-\frac{b s^{2} t_{a}}{\lambda_{0}+2 \lambda_{0}^{2} t_{a}} S^{\prime}(x) n^{0}\right) .
$$

Equation, (7.12) was derived for the simplified cartoon model (3.6), but a similar analysis can be done for the full cartoon model (2.2)-(2.3). This leads to the classical chemotaxis equation

$$
\frac{\partial n}{\partial t}=\frac{\partial}{\partial x}\left(\frac{s^{2}}{2 \lambda_{0}} \frac{\partial n}{\partial x}-g^{\prime}(S(x)) \frac{b s^{2} t_{a}}{\lambda_{0}\left(1+2 \lambda_{0} t_{a}\right)\left(1+2 \lambda_{0} t_{e}\right)} S^{\prime}(x) n\right)
$$

This is a parabolic counterpart of the hyperbolic equation (6.25). In particular, we derived again the formula for chemotactic sensitivity (6.26).

8. Numerical examples. The macroscopic descriptions of chemotaxis embodied in either the modified classical chemotaxis equation (6.24) or the classical chemotaxis equation (7.12) are approximations to the original transport equation and the stochastic process describing movement that underlies it. In this section we present two numerical examples that illustrate how well the macroscopic descriptions approximate the solution of the microscopic process. We start with the description of numerical methods which we use. 
8.1. Numerical methods. The parameters in our computations are assumed to be dimensionless, and we choose $b=1$, $t_{a}=1, \lambda_{0}=1$ and $s=0.1$, i.e., $s$ is small comparing to other parameters (compare with scaling (6.6) $-(6.7)$ ).

To solve the system (6.8) - (6.11), closed by (6.16), numerically, we first transform this system to the diagonal form

$$
\frac{\partial v}{\partial t}+D_{1} \frac{\partial}{\partial x} v=C_{1}(x) v
$$

Here $D_{1}$ is a diagonal $4 \times 4$ matrix. Then we use an explicit finite difference method with upwinding. To solve the hyperbolic modified chemotaxis equation (6.24) numerically, we first transform it to the system of two first order equations in the diagonal form

$$
\frac{\partial w}{\partial t}+D_{2} \frac{\partial}{\partial x} w=C(x) w
$$

where $D_{2}$ is a diagonal $2 \times 2$ matrix. Again, we use an explicit finite difference method with upwinding.

To solve the classical chemotaxis equation (7.12) numerically, we use an implicit finite difference method (backward difference approximation in time and centered difference approximation for spatial derivatives).

Finally, to simulate the random walk of individuals, we consider an ensemble of 2000 or 8000 particles, respectively. Each particle is described by three variables - position $x$, velocity $\pm s$ and the internal state $y$. We use a small time step $d t=0.01$ (i.e., the unbiased turning frequency divided by 100). During each time step the particle moves with speed $s$ in the chosen direction and we integrate the internal dynamics to find the change of $y$. At the end of each time step, a random number from $[0,1]$ is generated and compared with the probability of the turn $\lambda(y) \mathrm{d} t$. If the turn occurs, the bacterium will move during the next time step in the opposite direction. ${ }^{1}$

8.2. Traveling bands. In this example we analyze the motion of the individuals in the interval $[0,20]$ with the signal $S(x)$ given by

$$
S(x)=28-2|x-14| .
$$

The signal has a global maximum at the point 14 , and its derivative is $S^{\prime}(x)=-2 \operatorname{sign}(x-14)$ for $x \neq 14$. We assume the same initial condition for all computations, namely,

$$
n(x, 0)= \begin{cases}1 & \text { for } x \in[5,6] \\ 0 & \text { for } x \notin[5,6]\end{cases}
$$

we assume that all individuals are perfectly adapted at $t=0$, and we use no-flux boundary conditions.

In Figure 8.1 we compare the results of the stochastic simulation of the random walk with the solutions of the macroscopic system (6.8) - (6.11) closed by (6.16). It happens that the solution of the modified chemotaxis equation (6.24), and the solution of the classical chemotaxis equation (7.12) are indistinguishable on the plots from the solution of (6.8) - (6.11). Thus the macroscopic results presented can be viewed as plots of the solution of any of these macroscopic equations.

In Figure 8.1 we see that the band travels to the right (i.e., toward the maximum of the signal) as expected, and then the individuals who arrive at the maximum first aggregate there. Eventually all individuals aggregate around the maximum of the signal. From the plots we also see that numerically the macroscopic equations give very good results in comparison with the Monte Carlo simulations. Finally, if we use the results for the time interval $[0,600]$, i.e., under the influence of the constant gradient, we can compute the average speed of the bacteria in this interval, and find that

$$
V \doteq \frac{1}{150}=\frac{|b| s^{2} S^{\prime}(x) t_{a}}{\lambda_{0}+2 \lambda_{0}^{2} t_{a}}
$$

which agrees with the result in equation (5.7).

\footnotetext{
${ }^{1}$ A Monte Carlo simulation that incorporates the internal dynamics used here, as well as a more detailed description of the motor behavior, is given in [39].
} 

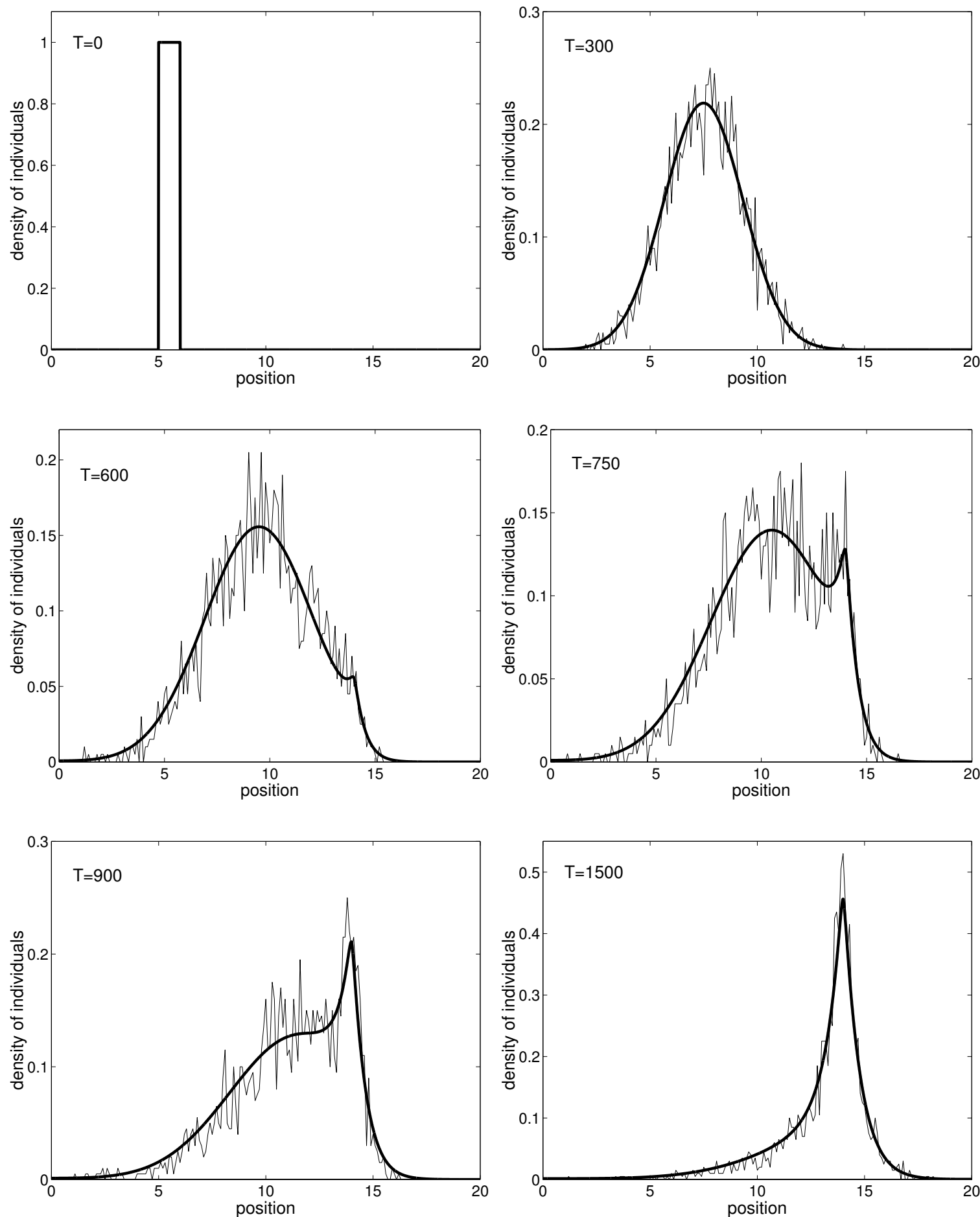

FIG. 8.1. The graphs show the solutions of the macroscopic system (6.8)-(6.11) closed by (6.16) (thick smooth line) and the results of stochastic simulations of the velocity jump process with internal state variables (thin line). Moreover, the thick line can also be viewed as a solution of the modified chemotaxis equations (6.24) and the solution of the classical chemotaxis equation (7.12), since the solutions of (6.24), (7.12) and (6.8) - (6.11) closed by (6.16) are indistinguishable on this scale. We used 2000 particles for the Monte Carlo simulations, and the parameters $b=1, t_{a}=1, \lambda_{0}=1$ and $s=0.1$. 

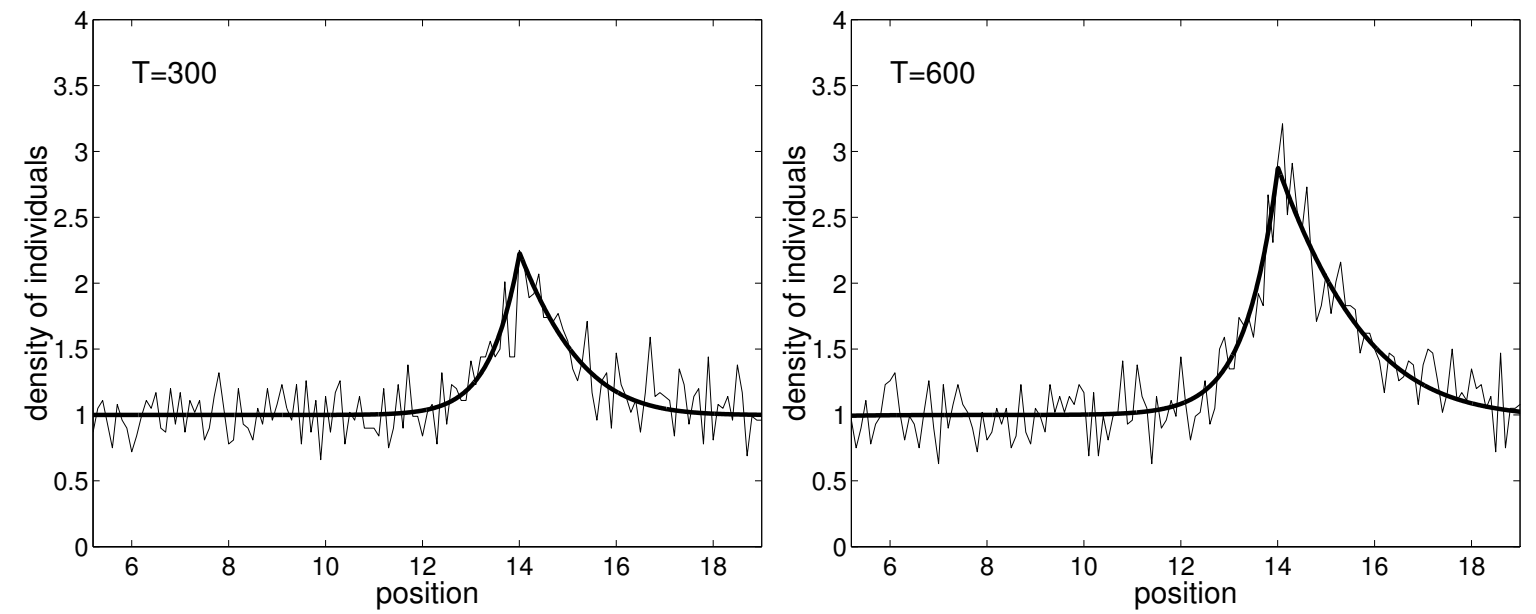

FIG. 8.2. The graphs of the density of bacteria under the influence of the exponential signal function (8.3) with $x_{0}=14$ and $C=1$. Initially the bacteria were uniformly distributed at a density equal to 1 . The figure shows the density of bacteria at time $T=300$ (left) and $T=600$ (right). The bacteria aggregate around the point $x=14$ as expected. In this figure we used the internal dynamics (2.2) $-(2.5)$ with $g(S)=2 \ln (S), t_{a}=b=\lambda_{0}=$ $1, t_{e}=0$ and $s=0.1$. The solution of the classical chemotaxis equation (7.12) (thick line) and the Monte Carlo simulation (thin line) are shown.

8.3. Exponential signal ramp. Various chemotaxis experiments have been done with exponential signal functions $[13,43]$. The standard setup is that initially there is a uniform concentration of bacteria in the medium with an exponential signal ramp of the form

$$
S(x)= \begin{cases}C e^{x} & x \leq x_{0} \\ C e^{x_{0}} & x \geq x_{0}\end{cases}
$$

After several minutes, bacteria aggregate at the top of the exponential ramp - i.e., around the point $x_{0}$.

As a second numerical example and test of the macroscopic equations, we reproduce these experiments with the exponential signal ramp. We again use here the internal dynamics (2.2) - (2.5) with a suitable choice of $g$. The exponential signal ramp was used experimentally because a cell swimming in one direction sees an constant rate of increase of the signal, and therefore the bias should remain approximately constant. To take this into account, we could choose

$$
g(S)=C \frac{S(x)}{K_{D}+S(x)} .
$$

However, as we only want to reproduce the experimental results qualitatively, we can approximate (8.4) by the logarithmic function. The numerical results for this are shown in Figure 8.2. We plot the solution of the classical chemotaxis equation (7.12), but the solutions of (6.8) - (6.11) and (6.24) again give the same results. Moreover, we also qualitatively reproduced the behavior observed in experiments $(c f .[13,43])$.

9. Extensions of the analysis. In this section, we discuss two extensions of our analysis - the inclusion of a finiteduration tumbling state and the moment closure for arbitrary signal gradients.

9.1. Inclusion of a finite-duration tumbling state. As we mentioned earlier, the movement of E.coli consists of "running" smoothly with a speed $s$ and "tumbling" randomly. Tumbles cause the bacterium to reorient and swim in a new random direction. The duration of both runs and tumbles are exponentially distributed with means of $1 \mathrm{sec}$ and $10^{-1}$ sec, respectively, in the absence of an extracellular signal. Thus cells spend 10 percent of the total time in the tumbling state. Earlier we neglected this time by assuming an instantaneous reversal of direction, but we now include it. We again restrict the analysis to one space dimension, since the generalization to higher dimensions only introduces some technical issues. We denote by

- $p^{0}(x, y, t) \ldots$ the number density of tumbling bacteria at time $t$ and at point $x$ with internal state $y$; 
- $p^{ \pm}(x, y, t) \ldots$ the number density of bacteria running to the right resp., left at time $t$ and at point $x$ with internal state $y$.

Suppose that a cell with internal state $y$ moves along the $x$-axis at a constant speed $s$, and at random instants of time stops with stopping time governed by a Poisson process of intensity $\alpha(y)$, and that cell with internal state $y$ tumbling at the point $x$ to move at random instants of time starts according to a Poisson process with the intensity $\beta(y)$. Further, suppose that the direction of movement is unbiased, i.e., that the tumbling particle will go with probability 0.5 to the right and with probability 0.5 to the left, given that movement starts.

For simplicity we consider the simplified cartoon internal dynamics (3.6) - (3.7). Using the change of internal variables $y_{2}=S(x)+z_{2}$, the movement of bacteria can be described by the following equations:

$$
\begin{gathered}
\frac{\partial p^{+}}{\partial t}+s \frac{\partial p^{+}}{\partial x}+\frac{\partial}{\partial z_{2}}\left[\left(-\frac{z_{2}}{t_{a}}-s S^{\prime}(x)\right) p^{+}\right]=-\alpha(y) p^{+}+\frac{1}{2} \beta(y) p^{0} \\
\frac{\partial p^{0}}{\partial t}+\frac{\partial}{\partial z_{2}}\left[\left(-\frac{z_{2}}{t_{a}}\right) p^{0}\right]=\alpha(y)\left(p^{+}+p^{-}\right)-\beta(y) p^{0} \\
\frac{\partial p^{-}}{\partial t}-s \frac{\partial p^{-}}{\partial x}+\frac{\partial}{\partial z_{2}}\left[\left(-\frac{z_{2}}{t_{a}}+s S^{\prime}(x)\right) p^{-}\right]=-\alpha(y) p^{-}+\frac{1}{2} \beta(y) p^{0} .
\end{gathered}
$$

In order to compare this model with the previous one, we will specify $\alpha(y)$ and $\beta(y)$ as follows

$$
\alpha(y)=2 \lambda_{0}+2 b z_{2}, \quad \beta(y)=\beta_{0}-\beta_{1} z_{2},
$$

where

$$
\lambda_{0}>0, \quad b>0, \quad \beta_{0}>0, \quad \beta_{1} \geq 0 .
$$

Then this model is equivalent to the model in Section 4 in the limit $\beta_{0} \rightarrow \infty$. One can show, using similar techniques as before, that the average position of the particles under the influence of a constant gradient $S^{\prime}(x)=C$ is given by (cf. (5.7))

$$
<x>(t)=\frac{b s^{2} C t_{a}}{\lambda_{0}+2 \lambda_{0}^{2} t_{a}} \frac{\beta_{0}}{2 \lambda_{0}+\beta_{0}} t .
$$

Thus the tumbling state slows down the movement by the factor $\beta_{0} /\left(2 \lambda_{0}+\beta_{0}\right)$ and we recover $(5.7)$ as $\beta_{0} \rightarrow \infty$. Moreover, one can derive the following modified classical chemotaxis equation ( $c f$. (6.24))

$$
\frac{\partial^{2} n}{\partial t^{2}}+2 \lambda_{0} \frac{\partial n}{\partial t}=\frac{\partial}{\partial x}\left(s^{2} \frac{\partial n}{\partial x}-\frac{2 s^{2} b t_{a}}{1+2 \lambda_{0} t_{a}} \frac{\beta_{0}}{2 \lambda_{0}+\beta_{0}} S^{\prime}(x) n\right) .
$$

9.2. Moment closure for arbitrary signal gradients. Heretofore we have used the approximation (6.16), which is appropriate for shallow signal gradients. The question arises as to what can be done for large signal gradients, i.e., for signals that satisfy the standing assumption (3.11). Can we also find a moment closure of the form (6.12)?

To do that, we have to approximate the neglected term

$$
2 b j_{2}=2 b s \int_{\mathbb{R}}\left(z_{2}\right)^{2}\left[p^{+}\left(x, z_{2}, t\right)-p^{-}\left(x, z_{2}, t\right)\right] \mathrm{d} z_{2}=2 b \int_{\mathbb{R}}\left(z_{2}\right)^{2} \mathcal{J}\left(x, z_{2}, t\right) \mathrm{d} z_{2} .
$$

Recall that the internal variable $z_{2} \in \mathbb{R}$ evolves according to (3.9), i.e., according to the differential equation

$$
\frac{\mathrm{d} z_{2}}{\mathrm{~d} t}=-\frac{z_{2}}{t_{a}} \mp S^{\prime}(x) s,
$$

where the sign of the last term is determined by the sign of the velocity of the particle. The equation (9.7) suggests that we can assume $z_{2} \approx \mp S^{\prime}(x) s t_{a}$. This is simply an assumption but it leads the following two naive moment closures:

$$
2 b j_{2}=2 b s \int_{\mathbb{R}}\left(z_{2}\right)^{2}\left[p^{+}\left(x, z_{2}, t\right)-p^{-}\left(x, z_{2}, t\right)\right] \mathrm{d} z_{2}=2 b s \int_{\mathbb{R}}\left(z_{2}\right)\left(z_{2}\right) p^{+}\left(x, z_{2}, t\right) \mathrm{d} z_{2}-2 b s \int_{\mathbb{R}}\left(z_{2}\right)\left(z_{2}\right) p^{-}\left(x, z_{2}, t\right) \mathrm{d} z_{2} \doteq
$$




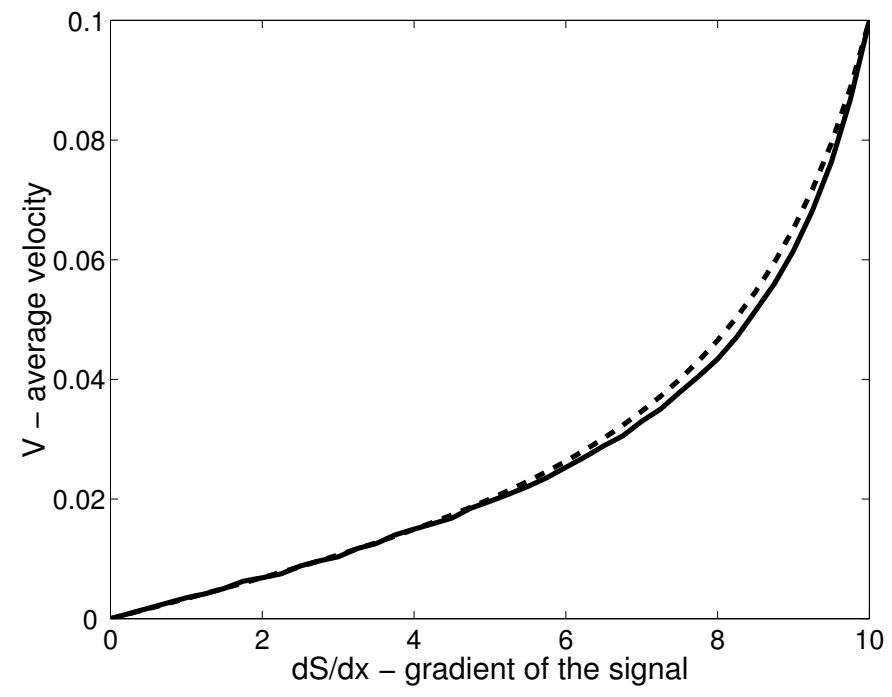

FIG. 9.1. The dashed line shows the average velocity $V$ given by the formula (9.10) as a function of the gradient of the signal. The solid line presents the average velocities of the bacteria obtained by the stochastic simulation.

$$
\begin{gathered}
\doteq 2 b s \int_{\mathbb{R}}\left(-S^{\prime}(x) s t_{a}\right)\left(z_{2}\right) p^{+}\left(x, z_{2}, t\right) \mathrm{d} z_{2}-2 b s \int_{\mathbb{R}}\left(S^{\prime}(x) s t_{a}\right)\left(z_{2}\right) p^{-}\left(x, z_{2}, t\right) \mathrm{d} z_{2}= \\
=-2 b S^{\prime}(x) t_{a} s^{2} \int_{\mathbb{R}} z_{2}\left[p^{+}\left(x, z_{2}, t\right)+p^{-}\left(x, z_{2}, t\right)\right] \mathrm{d} z_{2}=-2 b S^{\prime}(x) t_{a} s^{2} n_{1}, \\
2 b j_{2}=2 b \int_{\mathbb{R}}\left(z_{2}\right)^{2} \mathcal{J}\left(x, z_{2}, t\right) \mathrm{d} z_{2} \doteq 2 b \int_{\mathbb{R}}\left(\mp S^{\prime}(x) s t_{a}\right)^{2} \mathcal{J}\left(x, z_{2}, t\right) \mathrm{d} z_{2}=2 b\left(S^{\prime}(x)\right)^{2} t_{a}^{2} s^{2} j .
\end{gathered}
$$

These are both consistent with the moment closure (6.16) for shallow gradients of the signal, and consequently they lead to same equations (6.24) and (7.12) in that case. On the other hand they are much better than (6.16) for arbitrary signal gradients, which we illustrate here numerically.

To this end, suppose that the derivative of the signal function $S(x)$ is constant, i.e. $S^{\prime}(x)=C$ and, similarly as in Section 5, we derive the corresponding average velocity of the individuals $V$ (i.e. the average velocity which is approached asymptotically, $c f$. (5.7)). Surprisingly, the result is the same for both moment closures (9.8) and (9.9), namely

$$
V=\frac{b t_{a} s^{2} S^{\prime}(x)}{\lambda_{0}+2 \lambda_{0}^{2} t_{a}-2 b^{2} t_{a}^{3} s^{2}\left(S^{\prime}(x)\right)^{2}} .
$$

As in Section 8, we set $b=\lambda_{0}=t_{a}=1$ and $s=0.1$, and then have $\bar{C}=10$ and

$$
V=\frac{S^{\prime}(x)}{300-2\left(S^{\prime}(x)\right)^{2}},
$$

for $S^{\prime}(x) \in[-10,10]$. To verify this formula numerically, we make several stochastic simulations of the velocity jump process with internal variables, with the same parameters $b=\lambda_{0}=t_{a}=1$ and $s=0.1$ for the constant gradients of the signal from the interval $[0,10]$.

Figure 9.2 shows the graph of $V$ (dashed line) as a function of gradient $S^{\prime}(x)$. The solid line presents the velocities obtained by the stochastic simulation of the velocity jump process with internal variables. We see that for the maximum possible signal function $S^{\prime}(x)=10$, all individuals move to the right with the speed $s=0.1$ as expected. Moreover, we also see that the macroscopic equations obtained by the moment closure (9.8) or by moment closure (9.9) can give good macroscopic moment equations when used in (6.8) - (6.11). 
10. Discussion and conclusions. We have shown how information about microscopic intracellular processes such as signal transduction and response can be translated into the macroscopic chemotactic sensitivity that appears in the macroscopic description of chemotaxis. This was done for a highly-simplified description of the intracellular dynamics, one which is based on linear dynamics for the response to an extracellular signal, but which nonetheless incorporates the two most important characteristics of any detailed signal transduction network, namely, excitation and adaptation. Linear dynamics and linear response may well be adequate for describing the type of signal changes a swimming bacterium normally sees, but that remains to be established. In addition, a great deal of further work is needed to identify the essential response modes in a general signal transduction network, even if a near-equilibrium assumption is used. A difficult part of that will be to determine how the extracellular signal feeds into the linearized response of the cell.

The moment approach used here leads firstly to a system of hyperbolic equations, and then via a hyperbolic (resp., parabolic) scaling of space and time to a single hyperbolic (6.25) (resp., parabolic (7.13)) equation for the density of individuals. One can also use other scalings of space and time. For example the parabolic scaling uses $T \sim \mathcal{O}\left(1 / \varepsilon^{2}\right)$ and $L \sim \mathcal{O}(1 / \varepsilon)$, but if one use $T \sim \mathcal{O}\left(1 / \varepsilon^{3}\right)$, the resulting equation is the elliptic equation for the steady states of (6.25).

The first systematic derivation of a chemotaxis equation from a velocity jump process is due to Patlak [36], who considers both internal and external biases in detail, but these biases are imposed. A basic assumption in [36] is that the run length is chosen and fixed whenever the particle turns, which is quite different from the stochastic process treated here. As was observed elsewhere [30], the particle motion between turns is deterministic and thus, were the speed and run length constant, the process would be formally equivalent to a space jump process [28]. In general one can show that his process leads to a renewal equation that generalizes the renewal equation (15) derived in [28], from which a diffusion equation is obtained by suitable choice of the waiting time and jump distributions. Others have treated a processes similar to the one treated here without the internal dynamics since Patlak's work, and the reader is referred to [30] for a review of the literature.

\section{REFERENCES}

[1] W. ALT, Biased random walk models for chemotaxis and related diffusion approximations, J. Math. Biol., 9 (1980), pp. $147-177$.

[2] N. BARKAI AND S. LEIBLER, Robustness in simple biochemical networks, Nature, 387 (1997), pp. 913-917.

[3] J. Bartak, L. Herrmann, V. Lovicar, and O. Vejvoda, Partial differential equations of evolution, Ellis Horwood Limited, England, 1991.

[4] H. BERG, How bacteria swim, Scientific American, 233 (1975), pp. 36-44.

[5] - Random Walks in Biology, Princeton University Press, Princeton, NJ, USA, 1983.

[6] H. C. BERG, Bacterial microprocessing, Cold Spring Harbor Symp. Quantit. Biol., 55 (1990), pp. 539-545.

[7] H. C. BERG AND D. A. BROWN, Chemotaxis in esterichia coli analysed by three-dimensional tracking, Nature, 239 (1972), pp. $500-504$.

[8] S. M. Block, J. E. Segall, And H. C. BerG, Impulse responses in bacterial chemotaxis, Cell, 31 (1982), pp. $215-226$.

[9] - Adaptation kinetics in bacterial chemotactics, J. Bacteriology, 154 (1983), pp. 312-323.

[10] R. B. Bourret, K. A. Borkovich, And M. I. Simon, Signal transduction pathways involving protein phosphorylation in prokaryotes, Ann. Rev. Biochem., 60 (1991), pp. 401-441.

[11] C. Cercignani, The Boltzmann Equation and its Applications, Springer-Verlag, Berlin, Germany / Heidelberg, Germany / London, UK / etc., 1988.

[12] P. Cluzel, M. Surette, AND S. LeIBLER, An ultrasensitive bacterial motor revealed by monitoring signaling proteins in single cells, Science, 287 (2000), pp. 1652-1655.

[13] F. DAhlquist, P. Lovely, AND K. D. E., Quantitative analysis of bacterial migration in chemotaxis, Nature New Biology, 236 (1972), pp. 120123.

[14] S. Goldstein, On diffusion by discontinuous movements, and on the telegraph equation, Quarterly Journal of Mechanics and Applied Mathematics, VI (1951), pp. 129-156.

[15] G. J. HABETLER AND MATKOWSKY, Uniform asymptotic expansion in transport theory with small mean free paths, and the diffusion approximation, J. Math. Phy., 4 (1975), pp. 846-854.

[16] R. HERSH, Random evolutions: A survey of results and problems, Rocky Mountain J. Math., 4 (1974), pp. $443-477$.

[17] T. Hillen, Transport equations and chemosensitive movement, Habilitation thesis, University of Tuebingen, Germany, 2001.

[18] T. Hillen And H. OTHMER, The diffusion limit of transport equation derived from velocity-jump processes, Siam J. Appl. Math., 61 (2000), pp. 751-775.

[19] T. Hillen And A. Stevens, Hyperbolic models for chemotaxis in 1-D, Nonlinear Analysis: Real World Applications, 1 (2000), pp. 409-433.

[20] D. HoRstmAnn, From 1970 until present: the Keller-Segel model in chemotaxis and its consequences. (51 pages), preprint, 2003.

[21] M. KAC, A stochastic model related to the telegrapher's equation, Rocky Mountain J. Math., 3 (1974), pp. 497-509.

[22] E. KELLER AND L. SEGEL, Initiation of slime mold aggregation viewed as an instability, J. Theor. Biol, 26 (1970), pp. $399-415$.

[23] S. Khan, F. CAStellano, J. Spudich, J. MCCRAY, R. Goody, G. ReID, And D. Trentham, Excitatory signaling in bacteria probed by caged chemoeffectors, Biophys. J., 65 (1993), pp. 2368-2382.

[24] D. E. Koshland, Bacterial Chemotaxis as a Model Behavioral System, Raven Press, New York, NY, USA, 1980.

[25] E. W. LARSEn AND J. B. Keller, Asymptotic solution of neutron transport problems for small free mean paths, J. Math. Phys., 15 (1974), pp. 75-81.

[26] H. MCKEAN, Chapman-Enskog-Hilbert expansions for a class of solutions of the telegraph equation, Journal of Mathematical Physics, 75 (1967), pp. $1-10$. 
[27] C. J. Morton-Firth, T. S. Shimizu, And D. BRAY, A free-energy-based stochastic simulation of the Tar receptor complex, J Mol Biol, 286 (1999), pp. 1059-1074.

[28] H. Othmer, S. Dunbar, AND W. Alt, Models of dispersal in biological systems, Journal of Mathematical Biology, 26 (1988), pp. $263-298$.

[29] H. Othmer And T. Hillen, The diffusion limit of transport equations 2: Chemotaxis equations, Siam J. Appl. Math., 62 (2002), pp. 1222-1250.

[30] H. G. OTHMER AND T. HILlen, Chemotaxis equations from the diffusion limit of transport equations, SIAM JAM, 62 (2002), pp. $1222-1250$.

[31] H. G. OTHMER AND P. SCHAAP, Oscillatory cAMP signaling in the development of Dictyostelium discoideum, Comments on Theor. Biol., 5 (1998), pp. 175-282.

[32] H. G. OThmer AND A. STEVens, Aggregation, blowup, and collapse: The ABC's of taxis in reinforced random walks, SIAM Journal on Applied Mathematics, 57 (1997), pp. 1044-1081.

[33] K. J. PAinter, P. K. Maini, AND H. G. OThmer, Development and applications of a model for cellular response to multiple chemotactic cues. Submitted to J. Math Biol., 1999.

[34] G. C. Papanicolaou, Asymptotic analysis of transport processes, Bulletin AMS, 81 (1975), pp. 330-392.

[35] E. PATE AND H. G. OThmeR, Differentiation, cell sorting and proportion regulation in the slug stage of Dictyostelium discoideum, Jour. of Theor. Biol., 118 (1986), pp. 301-319.

[36] C. PATLAK, Random walk with persistence and external bias, Bull. Math: Biophys., 15 (1953), pp. 311-338.

[37] E. M. PuRCELL, Life at low Reynolds number, American Journal of Physics, 45 (1977), pp. 3-11.

[38] J. E. Segall, S. M. Block, AND H. C. BeRG, Temporal comparisons in bacterial chemotaxis, Proc. Nat. Acad. Sci. USA, 83 (1986), pp. 89878991.

[39] S. Setayeshgar, C. W. Gear, H. G. Othmer, And I. G. Kevrekidis, Application of coarse integration to bacterial chemotaxis. J. Chem. Phys., submitted.

[40] P. A. Spiro, J. S. Parkinson, And H. G. Othmer, A model of excitation and adaptation in bacterial chemotaxis, Proc. Nat. Acad. Sci. USA, 94 (1997), pp. 7263-7268.

[41] R. C. STEWART AND F. W. DAhlQuist, Molecular components of bacterial chemotaxis, Chem. Rev., 87 (1987), pp. 997-1025.

[42] J. B. Stock and M. G. Surette, Chemotaxis, in Escherichia coli and Salmonella: Cellular and Molecular Biology, F. C. N. et al., ed., vol. I, ASM Press, Washington, DC, 1996, pp. 1103-1129.

[43] R. M. WEIS AND D. E. KosHLAND, Reversible receptor methylation is essential for normal chemotaxis of Escherichia coli in gradients of aspartic acid, Proc. Nat. Acad. Sci., 85 (1988), pp. 83-87. 